\title{
Genome-wide identification and expression analysis of the ERF transcription factor family in pineapple (Ananas comosus (L.) Merr.)
}

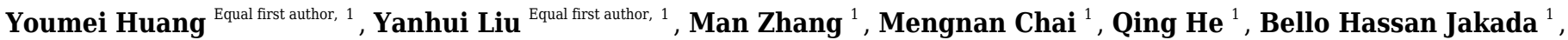 \\ Fangqian Chen ${ }^{1}$, Huihuang Chen ${ }^{1}$, Xingyue Jin ${ }^{1}$, Hanyang Cai ${ }^{\text {Corresp., }}{ }^{1}$, Yuan Qin ${ }^{\text {Corresp. } 1,2}$ \\ ${ }^{1}$ State Key Lab of Ecological Pest Control for Fujian and Taiwan Crops; Key Lab of Genetics, Breeding and Multiple Utilization of Crops, Ministry of \\ Education; Fujian Provincial Key Lab of Haixia Applied Plant Systems Biology, College of Life Sciences, Fujian Agriculture and Forestry University, Fuzhou, \\ Fujian Province, China \\ 2 State Key Laboratory for Conservation and Utilization of Subtropical Agro-Bioresources, Guangxi Key Lab of Sugarcane Biology, College of Agriculture, \\ Guangxi University, Nanning, Guangxi Province, China \\ Corresponding Authors: Hanyang Cai, Yuan Qin \\ Email address: caihanyang123@163.com, yuanqin@fafu.edu.cn
}

Pineapple (Ananas comosus (L.) Merr.) is an important tropical fruit with high economic value. The quality and yield of pineapple will be affected by various environmental conditions. Under adverse conditions, plants can produce a complex reaction mechanism to enhance their resistance. It has been reported that the member of ethylene responsive transcription factors (ERFs) plays a crucial role in plant developmental process and stress response. However, the function of these proteins in pineapple remains limited. In this study, a total of $74 E R F$ genes (AcoERFs) were identified in pineapple genome, named from ACOERF1 to ACOERF74, and divided into 13 groups based on phylogenetic analysis. We also analyzed gene structure, conserved motif and chromosomal location of AcoERFs, and the AcoERFs within the same group possess similar gene structures and motif compositions. 3 genes (ACoERF71, AcoERF73 and AcoERF74) were present on unanchored scaffolds, so they could not be conclusively mapped on chromosome. Synteny and cis-elements analysis of $E R F$ genes provided deep insight into the evolution and function of pineapple $E R F$ genes. Furthermore, we analyzed the expression profiling of $A c o E R F$ in different tissues and developmental stages, and 22 AcoERF genes were expressed in all examined tissues, in which 5 genes (AcoERF13, AcoERF16, AcoERF31, AcoERF42, and AcoERF65) had high expression levels. Additionally, 9 AcoERF genes were selected for functional verification by qRT-PCR. These results provide useful information for further investigating the evolution and functions of ERF family in pineapple. 
1 Genome-wide identification and expression analysis of

2 the ERF transcription factor family in pineapple

3 (Ananas comosus (L.) Merr.)

4

5

6

7

8

Youmei Huang ${ }^{1 \#}$, Yanhui Liu ${ }^{1 \#}$, Man Zhang ${ }^{1}$, Mengnan Chai ${ }^{1}$, Qing He ${ }^{1}$, Bello Hassan Jakada ${ }^{1}$, Fangqian Chen ${ }^{1}$, Huihuang Chen ${ }^{1}$, Xingyue Jin ${ }^{1}$, Hanyang Cai ${ }^{1 *}$, Yuan Qin $^{1,2 *}$

${ }^{1}$ State Key Lab of Ecological Pest Control for Fujian and Taiwan Crops; Key Lab of Genetics, Breeding and Multiple Utilization of Crops, Ministry of Education; Fujian Provincial Key Lab of Haixia Applied Plant Systems Biology, College of Life Sciences, Fujian Agriculture and Forestry University, Fuzhou, Fujian Province, China

${ }^{2}$ State Key Laboratory for Conservation and Utilization of Subtropical Agro-Bioresources, Guangxi Key Lab of Sugarcane Biology, College of Agriculture, Guangxi University, Nanning, Guangxi Province, China

Corresponding Author: Hanyang Cai ${ }^{1}$

No. 15, Shangxiadian Road, Fuzhou, Fujian Province 350002, China Email address: caihanyang123@163.com

Yuan Qin ${ }^{1,2}$

No. 15, Shangxiadian Road, Fuzhou, Fujian Province 350002, China

Email address: yuanqin@fafu.edu.cn

\section{ABSTRACT}

Pineapple (Ananas comosus (L.) Merr.) is an important tropical fruit with high economic value. The quality and yield of pineapple will be affected by various environmental conditions. Under adverse conditions, plants can produce a complex reaction mechanism to enhance their resistance. It has been reported that the member of ethylene responsive transcription factors (ERFs) plays a crucial role in plant developmental process and stress response. However, the function of these proteins in pineapple remains limited. In this study, a total of $74 E R F$ genes (AcoERFs) were identified in pineapple genome, named from AcoERF1 to AcoERF74, and divided into 13 groups based on phylogenetic analysis. We also analyzed gene structure, conserved motif and chromosomal location of AcoERFs, and the AcoERFs within the same group possess similar gene structures and motif compositions. 3 genes (AcoERF71, AcoERF73 and AcoERF74) were present on unanchored scaffolds, so they could not be conclusively mapped on chromosome. Synteny and cis-elements analysis of $E R F$ genes provided deep insight into the evolution and function of pineapple $E R F$ genes. Furthermore, we analyzed the expression profiling of $A c o E R F$ 
40

41

42

43

44

45

46

47

48

49

50

51

52

53

54

55

56

57

58

59

60

61

62

63

64

65

66

67

68

69

70

71

72

73

74

75

76

77

78

79

in different tissues and developmental stages, and $22 A c o E R F$ genes were expressed in all examined tissues, in which 5 genes (AcoERF13, AcoERF16, AcoERF31, AcoERF42, and $A c o E R F 65$ ) had high expression levels. Additionally, 9 AcoERF genes were selected for functional verification by qRT-PCR. These results provide useful information for further investigating the evolution and functions of ERF family in pineapple.

Keywords: pineapple, ERF transcription factors, phylogenetic analysis, synteny analysis, expression profiles

\section{INTRODUCTION}

Plant growth and development, yield and quality are frequently affected by unfavorable environmental factors such as drought, salinity, high temperature and cold. In order to survive these stress conditions, plants have developed complex reaction mechanisms at the molecular, cellular, and system levels (Mittler, 2006; Loudet \& Hasegawa, 2017). Gene co-expression at the transcriptional level is one of the most important ways to regulate biological processes.

Transcription factors (TFs) play important roles in regulating the expression of functional proteins when plants are exposed to unfavorable environmental conditions (Cui et al., 2016). Among these TFs, ethylene responsive transcription factors (ERFs) play important roles in various biological processes, such as defense responses and hormonal signal transduction (Agarwal et al., 2010; Sharma et al., 2010; Rashid et al., 2012). Therefore, it is important to study the evolution and function of these genes in order to improve yield and the ability to resist the adverse environmental conditions of plant.

The AP2/ERF super-family is one of the largest family in plant, and the member of this superfamily characterized by one or two conserved AP2/ERF domains of approximately 60 to 70 amino acids (Sakuma et al., 2002). It has been reported that the AP2/ERF domain contains tow conserved elements, YRG and RAYD element, and the core region of RAYD element has been predicted to form an amphipathic $\alpha$-helix (Okamuro et al., 1997). The three-dimensional (3D) structure of the AP2/ERF domain from AtERF1 shows that the domain is composed of a 3 antiparallel $\beta$-sheets and an $\alpha$-helix (Allen et al., 1998). Furthermore, tryptophan (Trp) and arginine (Arg) residues of the $\beta$-sheet are in contact with DNA during transcription (Sharma et al., 2010). Based on the number of AP2/ERF domains and other DNA binding domains, the AP2/ERF super-family can be divided into three families:AP2, ERF and RAV(Sakuma et al., 2002; Nakano et al., 2006). Among the three families, only the AP2 family contains two conserved AP2/ERF domains, whereas ERF and RAV family contain just a single AP2/ERF domain (Riechmann \& Meyerowitz, 1998). The members of the RAV family include an AP2/ERF domain and a B3domain (Romanel et al., 2009). Based on the ERF domain binding to DNA sequences, ERF family has been further classified into two major subfamilies: ERF (ethylene responsive transcription factors) subfamily and CBF/DREB (C-repeat binding factor/dehydration-responsive element binding factor) subfamily (Nakano et al., 2006). Proteins 
80

81

82

83

84

85

86

87

88

89

90

91

92

93

94

95

96

97

98

99

100

101

102

103

104

105

106

107

108

109

110

111

112

113

114

115

116

117

118

119

encoded by ERF subfamily genes bind to GCC box (AGCCGCC) (Ohmetakagi \& Shinshi, 1995; Hao et al., 1998), whereas the CBF/DREB subfamily genes typically binds to the DRE and/or Crepeat (A/GCCGAC) (Stockinger et al., 1997; Riechmann \& Meyerowitz, 1998). The residues at position 14 and 19 of ERF subfamily genes AP2/ERF domain is alanine (Ala) and aspartic acid (Asp), respectively, whereas DREB subfamily genes containing valine (Val) at position 14 and glutamine acid (Glu) at positions 19 (Sakuma et al., 2002).

ERFs are located downstream of the ethylene signaling pathway and regulate the transduction of ethylene as well as the trans-activation of certain transcription factors related to hormonal signal (Fujimoto et al., 2000; Sharma et al., 2010; Yu et al., 2012). In addition, ERF proteins are involved in various plant biological processes, such as environmental stresses response (Liu et al., 1999; Rashotte et al., 2006), beneficial symbiotic interaction(Vernie et al., 2008), as well as other developmental processes, such as leaf, flower and embryo development in some plants (Elliott et al., 1996; Moose \& Sisco, 1996; Boutilier et al., 2002; Vernie et al., 2008). To date, ERF family has been reported in various plant species, such as Arabidopsis thaliana (Sakuma et al., 2002; Nakano et al., 2006), soybean (Glycine max) (Li et al., 2005; Zhang et al., 2009), rice (Oryza sativa) (Cao et al., 2006; Nakano et al., 2006; Sharoni et al., 2011; Rashid et al., 2012), cotton (Gossypium barbadense L.) (Jin \& Liu, 2008; Meng et al., 2010), Populus trichocarpa (Zhuang et al., 2008), tomato (Solanum tuberosum L.) (Sharma et al., 2010; Pan et al., 2012), grape ((Vitis vinifera L.) (Licausi et al., 2010), cucumber (Cucumis sativus L.) (Hu \& Liu, 2011) and tartary buckwheat (Fagopyum Tataricum) (Liu et al., 2019).

Pineapple (Ananas comosus (L.) Merr.) is one of the most important tropical fruit in the world (Su et al., 2017; Xie et al., 2018; He et al., 2019). The growth and development of pineapple is affected by various environmental conditions, such as drought, salt and cold stress (He et al., 2019). Additionally, pineapple is also an important monocotyledonous, and it can be considered as a proper model to study the monocot evolution (Ming et al., 2015; He et al., 2019). Recently, a comprehensive study of the pineapple genome provide a solid foundation for the study of pineapple gene functions (Ming et al., 2015; Fang et al., 2016; Su et al., 2017). Due to the great economic and research value of pineapple, it would be meaningful to make a further study ERF family in pineapple.

Previous study only proposed the existence of DREB subfamily in the ERF family of pineapple (Chai et al., 2020). However, detail information about the whole ERF family members in pineapple remains unexplored. In order to investigate the function and evolution of other ERF family members in pineapple, we conducted a detailed analysis of the entire ERF family. In this study, 74 pineapple $E R F$ genes were identified and classified into 13 groups. Furthermore, we also conducted a systematic analysis including gene structure, motif compositions, chromosome distribution, phylogenetic and synteny analysis of each $A c o E R F$ gene. In order to further investigate the functions of $A c o E R F$ genes, we analyzed the expression profile of $A c o E R F$ genes 
120 in different tissues and stages. The data generated in this study will help to select the appropriate

121 candidate genes for further functional studies during pineapple growth and development. This is

122 of great significance to the investigation of pineapple stress response and variety improvement.

123

124 MATERIALS \& METHODS

\section{Genome-wide analysis of the ERF family in pineapple}

126 The AP2/ERF amino acid sequences of pineapple and Arabidopsis were downloaded from

127 Phytozome12 (https://phytozome.jgi.doe.gov/pz/portal.html) (Goodstein et al., 2012). The

128 Hidden Markov Model (HMM) profiles of the AP2 domain (PF00847) and the B3 domain

129 (PF02362) were obtained from PFAM database (http://pfam.sanger.ac.uk/) (Finn et al., 2008) as

130 the queries for search predicted AP2/ERF proteins in the pineapple dataset using HMMER

131 software 3.0 (http://hmmer.wustl.edu/) with a threshold of e-value $<\mathrm{e}^{-5}(E d d y, 2011)$. BLAST

132 searches were used to determine the predicted AP2/ERFs in pineapple database with all the

133 Arabidopsis AP2/ERFs as queries. All of the candidate genes were further examined by Simple

134 Modular Architecture Research Tool (SMART, http://smart.embl-heidelberg.de/) (Letunic \&

135 Bork, 2018) to confirm the protein sequences derived from the selected pineapple AP2/ERF

136 domains. ExPasy proteomics server (http://expasy.org/) (Gasteiger et al., 2003) was used to

137 predicate the basic information of $A c o E R F$ proteins, including molecular weight (MW) and

138 isoelectric points $(p I)$.

139

140

141

142

143

144

\section{Gene ontology (GO) annotation}

To investigate the putative function of the candidate $A c o E R F$ genes, the gene ontology (GO) annotations of the pineapple ERF family members were downloaded from Phytozome 12 (https://phytozome.jgi.doe.gov/pz/portal.html) (Goodstein et al., 2012).

\section{Sequence alignment and phylogenetic analysis}

Multiple sequence alignment of the AcoERF proteins was performed using DNAMAN (version

148

149 9) with the default parameters (Wang, 2015), and the diagram was visualized using ESPript 3.0 (http://espript.ibcp.fr/ESPript/cgi-bin/ESPript.cgi) (Gouet et al., 2003). To explore the evolution relationships of $E R F$ gene family members in pineapple, the ERF amino acid sequences from pineapple and Arabidopsis were used. The multiple sequence alignments of all ERF proteins were performed by using MUSCLE (http://www.ebi.ac.uk/Tools/msa/muscle/) (Edgar, 2004), with the default parameters. Subsequently, the phylogenetic trees were constructed using

153 MEGA6.0 software (http://www.megasoftware.net) (Tamura et al., 2013) via the Neighborjoining (NJ) method with the following parameters: node robustness was detected using the

155 bootstrap method, and the bootstrap was set to 1000 replications. Finally, the phylogenetic trees were generated by iTOL (https://itol.embl.de) (Letunic \& Bork, 2016).

\section{Gene structure and conserved motif predictions}

The exon/intron structures of $A c o E R F$ genes were obtained from the online program Gene 
160 Structure Display Server (GSDS, http://gsds.cbi.pku.edu.cn/) (Hu et al., 2015). The conserved

161 motifs in the AcoERF proteins sequences were investigated by Multiple EM for Motif Elicitation

162 (MEME, http://meme-suite.org/tools/meme) (Bailey et al., 2015). The optimized parameters

163 were as follows: maximum number of motifs was set to 20, the optimum width of motifs was set

164 to 10-50 residues, and other options were set to default.

165

166

167

\section{Chromosomal localization and synteny analysis}

168

169

The information of chromosome localization of all pineapple $E R F$ genes was obtained from Phytozome12 (https://phytozome.jgi.doe.gov/pz/portal.html). And then, each genes was mapped

170

171 on chromosomes using MapChart software based on positional information in the pineapple genome project (Voorrips, 2002). For synteny analysis, the sequence of rice and Arabidopsis

172 ERF proteins were downloaded from Phytozome12. The potential anchors within pineapple, between pineapple and Arabidopsis or rice were searched by BLASTP with the score value of

174 $\mathrm{E}<1 \mathrm{e}^{-5}$ and top 5 matches (Zhang et al., 2018). The syntenic block of ERFs within pineapple was visualized using the Circos Program (version 0.69) (http://circos.ca/) (Krzywinski et al., 2009).

176 The syntenic blocks between pineapple and 2 representative plants were constructed using J.

177 Craig Venter Institute (JCVI, https://github.com/tanghaibao/jcvi) (Tanenbaum et al., 2010). The

178 Synonymous $(K s)$ and non-synonymous $(K a)$ values of AcoERF sequences were calculated using 179 bio-pipeline-master (https://github.com/tanghaibao/bio- pipeline) (Tang et al., 2008).

181

\section{Expression analysis of $A c o E R F$ genes in different tissues}

182

183

To investigate the expression patterns of the $A c o E R F$ genes in different development and growth processes of pineapple, the RNA-Seq data of pineapple roots, leaves, flowers, fruits (stage 1-6) were downloaded from the online website Pineapple Genomics Database (PGD,

185 http://pineapple.angiosperms.org/pineapple/html/download.html) (Ming et al., 2015). The criterion of different stage of ovule, sepal, stamen and petal was referenced to the previously described methods (Su et al., 2017). Total RNA of these tissues was extracted using RNA extracted kit (Omega Bio-Tek, Shanghai, China) based on the supplier's instruction, and the samples were prepared based on the published method (Chen et al., 2017). The cDNA libraries for sequencing were constructed using the NEBNext Ultra ${ }^{\mathrm{TM}}$ RNA Library Prep Kit for Illumina

190

191 (NEB). The reads were aligned to pineapple genome by the TopHat 2.0.0 software with default

192 parameters (Trapnell et al., 2012). The Fragments Per Kilobase of transcript per Million fragments mapped (FPKM) value of each gene was calculated using Cuffdiff and Cufflinks

194

195 software with default parameters. Finally, a heatmap of AcoERF expression profile was produced by the heatmaply R package (Galili et al., 2018).

198

\section{Analysis of cis-elements from $A c o E R F$ promoters}

The promoter sequences of the AcoERF genes were downloaded from the Pineapple Genomics Database (http://pineapple.angiosperms.org/pineapple/html/index.html). And then, the stressrelated cis-elements of AcoERF genes were predicted using the Plant Cis-Acting Regulatory 
200 Element (PlantCARE, http://bioinformatics.psb.ugent.be/webtools/plantcare/html/) (Lescot et al.,

201 2002).

202

203

204

205

206

207

208

209

210

211

212

213

214

215

216

217

218

219

220

221

222

223

224

225

226

227

228

229

230

231

232

233

234

235

236

237

238

239

\section{Plant material and abiotic treatments}

The pineapple materials were provided by the Qin Lab (Center for Genomics and Biotechnology, Fujian Agriculture and Forestry University, Fujian, China). The seedings were grown in a greenhouse at $25^{\circ} \mathrm{C}, 16$-h light/8-h dark photoperiod and a relative humidity of $70 \%$ for one month. And then the one-month-old pineapple plants were exposed to the following treatments: cold stress $\left(4^{\circ} \mathrm{C}\right)$, drought stress $(350 \mathrm{mM}$ Mannitol) and salt stress $(150 \mathrm{mM} \mathrm{NaCl})$. The samples of pineapple roots and leaves were collected after 6, 12, 24 and $48 \mathrm{~h}$ treatments. The onemonth-old plants that without any stress treatment were used as controls in the expriment. The leaves and roots were harvested at the indicated time, and stored at $-80^{\circ} \mathrm{C}$ for subsequent analysis.

\section{RNA isolation and $q R T-P C R$ analysis}

Total RNA of the pineapple sample was extracted from roots and leaves using plant RNA extraction kit (OMEGA, Shanghai, China) according to the manufacturer's protocol. And then, total RNA was reverse-transcribed using AMV reverse transcriptase (Takara, Japan) based on the supplier's instruction. qRT-PCR analysis was conducted using SYBR Premix Ex Taq II system (Takara, Japan) and Bio-Rad Real-time PCR system (Foster city, CA, USA). The reactions were performed in a $20 \mu \mathrm{l}$ volume containing $10 \mu \mathrm{l}$ of $2 \times$ SYBR Premix, $8.2 \mu \mathrm{l}$ of RNase free water, $1 \mu \mathrm{l}$ of template, $0.4 \mu \mathrm{l}$ of each specific primer. The primers used for RT-PCR were showed in Table S1. The RT-PCR reactions program was completed with the following conditions: $95^{\circ} \mathrm{C}$ for $30 \mathrm{~s} ; 40$ cycles of $95^{\circ} \mathrm{C}$ for $5 \mathrm{~s}$ and $60{ }^{\circ} \mathrm{C}$ for $34 \mathrm{~s} ; 95^{\circ} \mathrm{C}$ for $15 \mathrm{~s}$ (Cai et al., 2017; Cai et al., 2019). The analyses were confirmed in triplicate. The relative expression level of each $A c o E R F$ gene was calculated based on the comparison threshold period $\left(2^{-\Delta \Delta C t}\right)$ method (Pan et al., 2012). The protein phosphatase $2 A$ gene from pineapple was used as the reference gene (Sang et al., 2013). The final data were subjected to an analysis of variance test.

\section{RESULTS}

\section{Genome-wide identification and characterization of pineapple ERF family}

A total of 103 pineapple genes were identified as possible encoding proteins containing AP2/ERF domain (Fig. 1A). Among these, 27 genes containing two conserved AP2/ERF domains and 2 genes possessed a single AP2/ERF domain together with a B3 domain were assigned to AP2 and RAV family, respectively; Remaining 74 genes with a single AP2/ERF domain were grouped into ERF family, including 20 DREB and 54 ERF subfamily members. Earlier, the DREB subfamily members of pineapple had been divided into 5 groups: I, II, III, IV and V (Chai et al., 2020). To maintain uniformity, all the pineapple ERF genes were provisionally named as AcoERF1-AcoERF74 according to the gene ID in ascending order (Table S2). The full-length of the 74 AcoERF proteins range from 105 (AcoERF36) to 605 (AcoERF17) amino acid residues, while the coding sequence (CDS) length ranges from 318 to 
$2401819 \mathrm{bp}$, and relative molecular weights (MW) range from 11.21 to $66.74 \mathrm{kDa}$. The predicted 241 isoelectric points ( $p I$ ) varied from 4.71 (AcoERF18 and AcoERF52) to 9.63 (AcoERF30) (Table 242 S2). Moreover, the Go annotation of AcoERFs included biological process (GO: 0006355) and 243 molecular function (GO: 0003700) (Table S2). Within biological process, 72 AcoERF genes 244 were associated with regulation of transcription and DNA-templated. For the molecular function 245 categories, $73 \mathrm{AcoERF}$ genes were involved in transcription factor activity and sequence-specific 246 DNA binding.

247

\section{Multiple sequence alignment and phylogenetic analysis of pineapple $E R F$ genes} Multiple sequence alignment of the 74 AcoERFs indicated that most ERF family members possessed conserved YRG and RAYD elements within the AP2/ERF domain region (Fig. S1).

252

253 ERF subfamily proteins containing Ala at position 14 and Asp at position19. However, the residues at position 14 and 19 of DREB subfamily proteins is Val and Glu, respectively. Evolution of ERF TFs was further explored based on the phylogenetic tree constructed with pineapple and Arabidopsis ERF genes (Fig. 1B). Earlier, seven Arabidopsis ERF proteins having low homology with other member of ERF family, were assigned to group VI-L and Xb-L according to the analysis of gene structure and conserved motif (Nakano et al., 2006). In this study, these seven Arabidopsis ERF proteins were also used for phylogenetic reconstruction and formed group $\mathrm{G}$ and L. The Arabidopsis ERF proteins of group I and III were from the same group in previous study (Nakano et al., 2006).Therefore, group I and III in this study were renamed as $\mathrm{C} 1$ and $\mathrm{C} 2$, respectively. The result showed that the AcoERFs were divided into 13 groups, except for AcoERF21. Group I contained the most AcoERFs, accounting for 16.22\% of total ERF genes. Followed by group J, which has 10 AcoERFs. However, group L has only one AcoERF.

\section{Gene structure and motif composition of pineapple ERF family}

To gain more insight into the evolution and structural diversity of the ERF family in pineapple, we analyzed the gene structure and conserved motif of the AcoERF genes. As shown in Fig. 2, 49 AcoERF genes have no intron, accounting for $66.22 \%$ of the total number of AcoERF genes, and all genes of group $\mathrm{J}$ have no intron. Moreover, the number of introns in these genes varied from 1 to 16, and most AcoERF genes have 1 to 3 introns, whereas AcoERF17 has the maximum number of introns. All genes of group $\mathrm{H}$ have 2 introns, except for AcoERF8. To further explore the divergence and functional relationship of AcoERF proteins, a total of 10 conserved motifs in the pineapple ERFs were identified by the MEME software, and the height of each letter in the logo was proportional to the conservation level of amino acid in all sequences analyzed (Fig. S2). As displayed in Fig. 3, motif 1 is present in every pineapple ERF protein, and almost all the proteins contain motif 2, except for AcoERF38 and AcoERF69. In addition, the motifs in different groups indicated that varying degrees of divergence among them. For example, motif 8

278 is unique to group H. Motif 6 is only present in group H and I. Motif 10 is only present in the 279 members of group $\mathrm{C} 1$ and $\mathrm{C} 2$. In general, the pineapple ERF proteins in the same group usually 
280

281

282

283

284

285

286

287

288

289

290

291

292

293

294

295

296

297

298

299

300

301

302

303

304

305

306

307

308

309

310

311

312

313

314

315

316

317

318

319

contained similar motifs, which indicates that they may play similar roles in the development and growth of pineapple.

\section{Chromosomal distribution and synteny analysis of $A c o E R F$ genes}

According to Fig. S3, $71 A c o E R F$ genes were unevenly distributed on the 23 pineapple linkage groups (LG). Among them, LG02 contains the most diverse AcoERF genes, accounting for $12.16 \%$ of total genes, whereas LG04, LG14, LG19, and LG25 possessed only one AcoERF gene, respectively. Additionally, AcoERF71, AcoERF73 and AcoERF74 exist on unanchored scaffolds, so they could not be conclusively charted on any pineapple linkage groups (Table S2).

Segmental duplication provide an important mechanism for the expansion of gene families (Lynch \& Conery, 2000; Vision et al., 2000; Cannon et al., 2004; Zhang et al., 2018). A total of 16 segmental duplication evens with $24 A c o E R F$ genes were found from pineapple genome, such as AcoERF13/AcoERF5, AcoERF16/AcoERF31,AcoERF19/AcoERF35, all of these were segmental duplicates (Fig. 4, Table S3), and these segmental duplication pairs play crucial roles in the expansion of ERF family in pineapple genome. In order to further study the phylogenetic and evolutionary trait of ERF family in pineapple, we set up 2 comparative syntenic maps of pineapple associated with 2 representative plant, including Arabidopsis and rice (Fig. 5A, B). According to the syntenic results, there are 37 and 44 orthologous gene pairs in Arabidopsis and rice, respectively (Table S4-S5). Some AcoERF genes had multiple orthologous gene pairs (one pineapple gene associated with multiple Arabidopsis or rice genes), such as AcoERF62 associated with AtERF008/019/020, and AcoERF29 associated with OsERF025/026/027, suggesting these genes might play crucial roles in the evolution of ERF family. Some $A c o E R F$ genes could be found orthologous gene pairs in Arabidopsis and rice, such as AcoERF15, $A c o E R F 22$ and AcoERF32, suggesting that these genes might already exist before speciation. Some $A c o E R F$ genes only existed orthologous gene pairs between pineapple and rice, such as AcoERF15, AcoERF22 and AcoERF32, suggesting that the appearance of these orthologous gene pairs before the divergence of monocotyledonous and dicotyledonous plants. In order to further understand the evolution of $E R F$ gene family, the $K a / K s$ ratios of the $E R F$ gene pairs were calculated. The $K a / K s$ ratio can represent different selection categories for duplication genes, such as the $K a / K s>1$ indicates positive selection, the $K a / K s=1$ indicates neutral evolution, and the $K a / K s<1$ indicates negative selection (Li et al., 2019). According to the results, the $K a / K s$ ratio of most orthologous $E R F$ gene pairs are less than 1, indicating that they might have experienced strong negative selection pressure during the evolution of pineapple.

\section{Expression patterns of $A c o E R F$ genes in different tissues of pineapple}

The expression profiles of $74 A c o E R F$ genes in various pineapple tissues at different development stages was performed using RNA-seq expression data from MD2 pineapple plants recently published (Ming et al., 2015). According to the result, a total of $70 A c o E R F$ genes were expressed in different tissues, whereas 4 AcoERF genes (AcoERF22, AcoERF32, AcoERF43, 
320 AcoERF72) were not found in the RNA-seq libraries (Fig. 6, Table S6). 22 genes were expressed

321 in all tested tissues, in which 5 genes (AcoERF13, AcoERF16, AcoERF31, AcoERF42,

322 AcoERF65) showed relative high expression levels (value $>10$ ), suggesting these genes might

323 played the indispensable roles in pineapple development. Moreover, 8, 16, 6, 9 genes showed

324 high expression levels at all selected stages in ovule, sepal, stamen and petal, respectively; 16, 13,

32512 genes showed high expression levels in leaf, flower and root, respectively; 10 genes showed

326 high expression levels in fruit at all detected stages, indicating that these genes might be involved

327 in the growth of relative tissues. Additionally, AcoERF42 and AcoERF65 showed the highest

328 expression levels in fruit, suggesting that these genes might play positive roles in the growth and

329 development of fruit.

330

331

332

333

334

335

336

337

338

339

340

341

342

\section{Identification of cis-elements in AcoERF promoters}

In order to determine the response mechanisms of AcoERFs, 13 stress-related cis-elements were found in pineapple ERF promoters, such as low temperature response elements (LTR), dehydration responsive elements (DRE) and defense and stress responsive elements (TC-rich) (Table S7). LTRs are involved in low-temperature stress response (Choudhury et al., 2008); DRE and TC-rich repeats play roles in dehydration, low temperature, and salt stress response (Diazdeleon et al., 1993; Germain et al., 2012).According to the results, all AcoERF genes contain more than two cis-elements in their promoters. The promoter of AcoERF23 only contained two cis-elements, whereas the most ten in the promoter of AcoERF46. In addition, at least one ABRE was present in 85.13\% (63 out of 74) AcoERF gene promoters. The analysis of the 13 cis-elements suggested that most of AcoERF genes could response to different stress conditions.

343

\section{Expression profiles of $\boldsymbol{A c o E R F}$ genes under various abiotic stresses}

345 According to previous studies, some $E R F$ genes were involved in abiotic stress response in various plants, such as Arabidopsis (Sakuma et al., 2002), grape (Zhuang et al., 2009), tomato (Sharma et al., 2010), peach (Amygdalus persica L.) (Zhang et al., 2012). However, there was no report about the function of ERF genes in response to abiotic stress in pineapple. To investigate the roles of AcoERF genes in abiotic stress response, 9 AcoERF genes in roots and leaves were selected for functional verification. RT-PCR experiments were performed to analyze the expression patterns in response to different abiotic stress treatments, including cold, drought and salt stress (Fig. 7-9, Table S8-S10). Overall, we found that the expression of these genes was influenced by the treatments. Among these treatments, some AcoERF genes in different pineapple tissues were significantly induced by the treatments, such as AcoERF36 AcoERF45,

355

356 and AcoERF67. Interestingly, the expression patterns of AcoERF genes from root and leaf were

357 different under the same treatments, such as AcoERF13, AcoERF16 and AcoERF42 under cold stress, AcoERF7, AcoERF36 and AcoERF60 in response to drought and salt stress, indicated that these genes may have different functions in various tissues of pineapple. Under cold and drought stress, the expression level of most AcoERF genes in leaves was higher than that in roots, while 
360 it was the opposite under salt stress, suggesting that $A c o E R F$ genes in root were sensitive to salt

361

362

363

364

365

366

367

368

369

370

371

372

373

374

375

376

377

378

379

380

381

382

383

384

385

386

387

388

389

390

391

392

393

394

395

396

397

398

399

stress. Besides, most of $A c o E R F$ genes had maximal expression before 12 hours in drought and salt stress, indicating these genes might play key roles in the early stage of drought and salt stress responses.

\section{DISCUSSION}

The ERF family is one of the most important transcription factor families, and it belongs to AP2/ERF super-family, which also contains AP2 and RAV family (Sakuma et al., 2002; Nakano et al., 2006). It has been reported that the member of ERF family plays a crucial role in the growth and development of various plants (Fits \& Memelink, 2000; Banno et al., 2001; Yu et al., 2012; Muller \& Munnebosch, 2015). Previous studies have identified 120 ERF family members in soybean (Li et al., 2005), 103 in cucumber (Hu \& Liu, 2011), 85 in tomato (Sharma et al., 2010), 139 and 122 in rice and Arabidopsis, respectively (Nakano et al., 2006; Sharoni et al., 2011), but the identification of pineapple ERF gene family has not been reported. Pineapple has great economic and research value, so it is necessary to investigate the function of ERF family in pineapple. In this study, 103 candidate $A P 2 / E R F$ genes were identified in pineapple genome, including 27 AP2 family members, 2 RAV family members and 74 ERF family members (Fig. 1A). Compared with soybean, cucumber, tomato, rice and Arabidopsis, the pineapple ERF family is relatively small, indicating that some ERF members of pineapple may be lost during the evolution of species. To reveal the phylogenetic relationship of pineapple ERF family, a phylogenetic tree was constructed, 74 pineapple ERF family members were divided into 13 groups (Fig. 1B).

Gene structure analysis plays a crucial role in revealing the function of genes. Here, our results suggested that $A c o E R F s$ possess introns with number varying from 0 to 16, and the ERF members within the same group exhibited similar gene structure (Fig. 2). $66.22 \% A c o E R F$ genes had no introns, which is similar to the status in Arabidopsis, cucumber and tartary buckwheat (Nakano et al., 2006; Hu \& Liu, 2011; Liu et al., 2019). Presence of long, multiple intros can delay transcriptional output, which may suppress the expression of genes under adverse conditions. Conversely, the genes with small or fewer introns may have efficient expression in response to stress environments (Jeffares et al., 2008; Heyn et al., 2015). Hence, a large number of intron-less $A c o E R F$ genes may react rapidly when the external environment changes. For instance, AcoERF13, AcoERF36, AcoERF45 and AcoERF67 had no introns, and they had efficient expression under various stresses within 48h (Fig. 7-9, Table S8-S10).

The domains and motifs of transcription factors play essential roles in proteins interaction, transcriptional activity and DNA binding (Liu et al., 1999). Here, a total of 10 conserved motifs in the pineapple ERFs were identified (Fig. 3). Different types and numbers of motifs in the pineapple ERF proteins could affect the diversity of gene function. Motif 1,2 and 3 correspond to the AP2/ERF domain region, and were highly conserved in pineapple ERF family members.

Peer) reviewing PDF | (2020:04:47897:2:0:NEW 12 Aug 2020) 
400 Motif 4 is present in several AocERFs and specifically rich in glutamine (Q). The presence of

401

402

403

404

405

406

407

408

409

410

411

412

413

414

415

416

417

418

419

420

421

422

423

424

425

426

427

428

429

430

431

432

433

434

435

436

437

438 poly (Q) motif is related to protein aggregation and can stabilize protein-protein interactions ( $\mathrm{Liu}$ et al., 2011; Schaefer et al., 2012). Motif 5 containing multiple serine residues, and the poly serine repeats role as flexible linker, as well as an important site of several posttranslational modifications (Uversky, 2015). Therefore, AcoERFs containing motif 5 may paly crucial roles in various pathways. Motif 8 is specifically detected in group H, and the "MCGGAI" residues were highly conserved in the N-terminal region of the motif 8 . It has been reported that the overexpression of the ERF genes with the "MCGGAI" motif can improve plant tolerance under hypoxia stress ( $X u$ et al., 2006). Besides, the genes containing "MCGGAI" motif is also involved in ethylene transcription activation (Buttner \& Singh, 1997). Although the function of some conserved motifs is still unknown, they may also be involved in transcriptional regulation. In general, the ERF family members within the same group shared similar gene structures and motif compositions, which suggests that they may have similar roles in plant development and growth.

Segmental duplication occurs frequently in plants since most plants are diploidized polyploids (Zhu et al., 2014). In this study, a total of 16 segmental duplication evens with 24 AcoERF genes were found from pineapple genome (Fig. 4, Table S3). The diversity of gene combinations may be one of the factors that make the regulatory relationship complicated (Zhang et al., 2018; Zhang et al., 2020). Polyploidization, a widespread phenomenon among plant, is considered an important process of plant speciation and evolution, and the formation of polyploid includes hybridization and genomic doubling process before or after hybridization (Kohler et al., 2010; Zeng et al., 2020). Various Arabidopsis and rice ERF genes have been reported to be involved in the regulation of plant stress tolerance. For instance, At5g52020 and OsDREB 1F have been shown to play crucial roles in high-salt, low-temperature and drought stresses (Nakano et al., 2006). The synteny analysis could reveal the functional and evolutional connections between two species. Here, 15 pineapple ERF genes and 25 Arabidopsis ERF genes, 23 pineapple and 32 rice $E R F$ genes were identified as orthologous gene pairs, and different kinds of syntenic orthologous gene pairs could be investigated the evolutionary process of ERF family members in pineapple (Fig. 5A, B, Table S4-S5). Some ERF genes did not find any orthologous gene pairs, which can be attributed to the rearrangement or fusion of chromosomes during their evolution (He et al., 2014; Zhang et al., 2018).

The analysis of gene expression patterns can be preliminarily predicted the function of genes (Peng et al., 2015; Su et al., 2017; Zhang et al., 2018). In this study, 5 genes (AcoERF13, AcoERF16, AcoERF31,AcoERF42, AcoERF65) showed high expression levels in all selected pineapple tissues (Fig. 6, Table S6), indicating that $E R F$ genes may play a crucial role in pineapple development. Some $A c o E R F$ genes showed high expression levels in fruit, such as AcoERF16, AcoERF45, AcoERF62, suggesting that these genes play important roles in fruit ripening. Moreover, some $A c o E R F$ genes are expressed in various tissues or diverse stages, 
439 indicating that these genes could be more stable than those that only expressed in specific tissues

440

441

442

443

444

445

446

447

448

449

450

451

452

453

454

455

456

457

458

459

460

461

462

463

464

465

466

467

468

469

470

471

472

473

474

475

476

477

478

or one stage of an organ (He et al., 2019).

When plants encounter stress conditions, a series of cell activities and molecules reaction mechanisms can improve the resistance of plants (Chinnusamy et al., 2004; Mittler, 2006). According to previous studies, some $E R F$ genes were involved in various stresses responses in plants, such as high-salt, low-temperature and drought stress (Ohmetakagi \& Shinshi, 1995; Sakuma et al., 2002; Nakano et al., 2006). The expression profile of AcoERF genes suggested that several genes may be involved in the mechanism of abiotic stresses response (Fig. 7-9, Table S8-S10). Besides, AcoERF16, AcoERF42 and AcoERF65 came from the same group H, and AcoERF16 and AcoERF65 was segmental duplication gene pair. Although these three genes had similar gene structure and motif compositions, the expression patterns of AcoERF16 and AcoERF 65 were more similar under different treatments. The differential expression indicated that segmental duplication might influence the expression of gene. Overall, the above findings provide foundation to further investigate the potential function of pineapple $E R F$ genes. These analyses are not only helpful in selecting valuable candidate $E R F$ genes for further functional studies but also has important implication for genetic improvement for agricultural production and stress tolerance in pineapple crop.

\section{CONLUSIONS}

Based on genomic data of pineapple, a genome-wide identification of pineapple ERF family was performed, and 74 AcoERFs were identified. A comprehensive analysis of their phylogenetic relationships, gene structures and conserved motifs compositions showed high levels of similarity within the same group. Synteny analysis of ERF genes revealed the evolutionary characteristic of pineapple ERF family. The expression profile of AcoERFs verified their roles in responding to various abiotic stresses (cold, drought and salt stress). These results will help to further study the $E R F$ genes and their role in abiotic and biotic stress tolerance for improve the agricultural productivity of pineapple crop.

\section{ACKNOWLEDGEMENTS}

We would like to thank the reviewers for their helpful comments on the original manuscript. This work was supported by the National Natural Science Foundation of China (31761130074, 31970333 and U1605212 to Yuan Qin; 31700279 to Hanyang Cai) and a Guangxi Distinguished Experts Fellowship to Yuan Qin. The funders had no role in the study design, data collection and analysis, decision to publish or preparation of the manuscript.

\section{REFERENCES}

\section{Agarwal P, Agarwal PK, Joshi AJ, Sopory SK, Reddy MK. 2010. Overexpression of} PgDREB2A transcription factor enhances abiotic stress tolerance and activates downstream stress-responsive genes. Molecular Biology Reports 37:1125-1135. 
479

480

481

482

483

484

485

486

487

488

489

490

491

492

493

494

495

496

497

498

499

500

501

502

503

504

505

506

507

508

509

510

511

512

513

514

515

516

517

518

10.1007/s11033-009-9885-8

Allen M, Yamasaki K, Ohmetakagi M, Tateno M, Suzuki M. 1998. A novel mode of DNA recognition by a beta-sheet revealed by the solution structure of the GCC-box binding domain in complex with DNA. The EMBO journal 17:5484-5496. 10.1093/emboj/17.18.5484

Bailey TL, Johnson JR, Grant CE, Noble WS. 2015. The MEME Suite. Nucleic acids research 43:39-49. 10.1093/nar/gkv416

Banno H, Ikeda Y, Niu Q, Chua N. 2001. Overexpression of Arabidopsis ESR1 induces initiation of shoot regeneration. The Plant cell 13:2609-2618. 10.1105/tpc.010234

Boutilier K, Offringa R, Sharma VK, Kieft H, Ouellet T, Zhang L, Hattori J, Liu C, Lammeren AAMV, Miki B, Custers JBM, Campagne MMVL. 2002. Ectopic expression of BABY BOOM triggers a conversion from vegetative to embryonic growth. The Plant cell 14:1737-1749. 10.1105/tpc.001941

Buttner M, Singh K. 1997. Arabidopsis thaliana ethylene-responsive element binding protein (AtEBP), an ethylene-inducible, GCC box DNA-binding protein interacts with an ocs element binding protein. Proceedings of the National Academy of Sciences of the United States of America 94:5961-5966. 10.1073/pnas.94.11.5961

Cai H, Zhang M, Chai M, He Q, Huang X, Zhao L, Qin Y. 2019. Epigenetic regulation of anthocyanin biosynthesis by an antagonistic interaction between H2A.Z and H3K4me3. New Phytologist 221:295-308. 10.1111/nph.15306

Cai H, Zhao L, Wang L, Zhang M, Su Z, Cheng Y, Zhao H, Qin Y. 2017. ERECTA signaling controls Arabidopsis inflorescence architecture through chromatin - mediated activation of PRE1 expression. New Phytologist 214(4):1579-1596. 10.1111/nph.14521

Cannon SB, Mitra A, Baumgarten A, Young ND, May G. 2004. The roles of segmental and tandem gene duplication in the evolution of large gene families in Arabidopsis thaliana. BMC Plant Biol 4:10. 10.1186/1471-2229-4-10

Cao Y, Song F, Goodman R, Zheng Z. 2006. Molecular characterization of four rice genes encoding ethylene-responsive transcriptional factors and their expressions in response to biotic and abiotic stress. Plant Physiol 163:1167-1178. 10.1016/j.jplph.2005.11.004

Chai M, Cheng H, Yan M, Priyadarshani S, Zhang M, He Q, Huang Y, Chen F, Liu L, Huang X, Lai L, Chen H, Cai H, Qin Y. 2020. Ananas comosusIdentification and expression analysis of the DREB transcription factor family in pineapple ( (L.) Merr.). PeerJ 8:e9006. 10.7717/peerj.9006

Chen P, Li Y, Zhao L, Hou Z, Yan M, Hu B, Liu Y, Azam S, Zhang Z, Rahman Z, Liu L, Qin Y. 2017. Ananas comosusGenome-Wide Identification and Expression Profiling of ATP-Binding Cassette (ABC) Transporter Gene Family in Pineapple ( (L.) Merr.) Reveal the Role of in Pollen Development. Frontiers in plant science 8:2150. 10.3389/fpls.2017.02150

Peer] reviewing PDF | (2020:04:47897:2:0:NEW 12 Aug 2020) 
519 Chinnusamy V, Schumaker K, Zhu J. 2004. Molecular genetic perspectives on cross-talk

520

521

522

523

524

525

526

527

528

529

530

531

532

533

534

535

536

537

538

539

540

541

542

543

544

545

546

547

548

549

550

551

552

553

554

555

556

557

558 and specificity in abiotic stress signalling in plants. Journal of experimental botany 55:225-236. 10.1093/jxb/erh005

Choudhury SR, Roy S, Das R, Sengupta D. 2008. Differential transcriptional regulation of banana sucrose phosphate synthase gene in response to ethylene, auxin, wounding, low temperature and different photoperiods during fruit ripening and functional analysis of banana SPS gene promoter. Planta 229:207-223. 10.1007/s00425-0080821-2

Cui L, Feng K, Wang M, Wang M, Deng P, Song W, Nie X. 2016. Genome-wide identification, phylogeny and expression analysis of AP2/ERF transcription factors family in Brachypodium distachyon. Bmc Genomics 17:1-19. 10.1186/s12864-0162968-8

Diazdeleon F, Klotz KL, Lagrimini L. 1993. Nucleotide sequence of the tobacco (Nicotiana tabacum) anionic peroxidase gene Plant Physiol 101:1117-1118. 10.1104/pp.101.3.1117

Eddy SR. 2011. Accelerated Profile HMM Searches. PLoS computational biology 7:e1002195. 10.1371/journal.pcbi.1002195

Edgar RC. 2004. MUSCLE: multiple sequence alignment with high accuracy and high throughput. Nucleic acids research 32:1792-1797. 10.1093/nar/gkh340

Elliott R, Betzner A, Huttner E, Oakes M, Tucker W, Gerentes D, Perez P, Smyth D. 1996. AINTEGUMENTA, an APETALA2-like gene of Arabidopsis with pleiotropic roles in ovule development and floral organ growth. The Plant cell 8:155-168. 10.1105/tpc.8.2.155

Fang J, Miao C, Chen R, Ming R. 2016. Genome-Wide Comparative Analysis of Microsatellites in Pineapple. Tropical Plant Biology 9:117-135. 10.1007/s12042016-9163-6

Finn R, Tate J, Mistry J, Coggill P, Sammut S, Hotz H, Ceric G, Forslund K, Eddy S, Sonnhammer ELL, Bateman A. 2008. The Pfam protein families database. Nucleic acids research 36:D281-D288. 10.1093/nar/gkm960

Fits LVD, Memelink J. 2000. ORCA3, a jasmonate-responsive transcriptional regulator of plant primary and secondary metabolism. Science 289:295-297. 10.1126/science.289.5477.295

Fujimoto S, Ohta M, Usui A, Shinshi H, Ohmetakagi M. 2000. Arabidopsis ethyleneresponsive element binding factors act as transcriptional activators or repressors of GCC box-mediated gene expression. The Plant cell 12:393-404. 10.1105/tpc.12.3.393

Galili T, Ocallaghan A, Sidi J, Sievert C. 2018. heatmaply: an R package for creating interactive cluster heatmaps for online publishing. Bioinformatics 34(9):1600-1602. DOI 10.1093/bioinformatics/btx657

Gasteiger E, Gattiker A, Hoogland C, Ivanyi I, Appel RD, Bairoch A. 2003. ExPASy: The

Peer) reviewing PDF | (2020:04:47897:2:0:NEW 12 Aug 2020) 
559

560

561

562

563

564

565

566

567

568

569

570

571

572

573

574

575

576

577

578

579

580

581

582

583

584

585

586

587

588

589

590

591

592

593

594

595

596

597

598

proteomics server for in-depth protein knowledge and analysis. Nucleic acids research 31:3784-3788. 10.1093/nar/gkg563

Germain H, Lachance D, Pelletier G, Fossdal CG, Solheim H, Séguin A. 2012. The expression pattern of the Picea glauca Defensin 1 promoter is maintained in Arabidopsis thaliana, indicating the conservation of signalling pathways between angiosperms and gymnosperms. Journal of experimental botany 63:785-795. 10.1093/jxb/err303

Goodstein D, Shu S, Howson R, Neupane R, Hayes R, Fazo J, Mitros T, Dirks W, Hellsten U, Putnam N, Rokhsar D. 2012. Phytozome: a comparative platform for green plant genomics. Nucleic acids research 40:1178-1186. 10.1093/nar/gkr944

Gouet P, Robert X, Courcelle E. 2003. ESPript/ENDscript: Extracting and rendering sequence and 3D information from atomic structures of proteins. Nucleic acids research 31:3320-3323. 10.1093/nar/gkg556

Hao D, Ohmetakagi M, Sarai A. 1998. Unique mode of GCC box recognition by the DNAbinding domain of ethylene-responsive element-binding factor (ERF domain) in plant. Journal of Biological Chemistry 273:26857-26861. 10.1074/jbc.273.41.26857

He D, Lei Z, Xing H, Tang B. 2014. Genome-wide identification and analysis of the aldehyde dehydrogenase (ALDH) gene superfamily of Gossypium raimondii. Gene 549:123-133. 10.1016/j.gene.2014.07.054

He Q, Liu Y, Zhang M, Bai M, Priyadarshani SVGN, Chai M, Chen F, Huang Y, Liu L, Cai H, Qin Y. 2019. Genome-Wide Identification and Expression Analysis of the NAC Transcription Factor Family in Pineapple. Tropical Plant Biology 12:255-267. 10.1007/s12042-019-09233-3

Heyn PC, Kalinka A, Tomancak P, Neugebauer K. 2015. Introns and gene expression: cellular constraints, transcriptional regulation, and evolutionary consequences. BioEssays 37:148-154. 10.1002/bies.201400138

Hu B, Jin J, Guo A, Zhang H, Luo J, Gao G. 2015. GSDS 2.0: an upgraded gene feature visualization server. Bioinformatics 31:1296-1297. 10.1093/bioinformatics/btu817

Hu L, Liu S. 2011. Genome-wide identification and phylogenetic analysis of the ERF gene family in cucumbers. Genetics and Molecular Biology 34:624-634. 10.1590/s141547572011005000054

Jeffares D, Penkett C, Bahler J. 2008. Rapidly regulated genes are intron poor. Trends in Genetics 24:375-378. 10.1016/j.tig.2008.05.006

Jin L, Liu J. 2008. Molecular cloning, expression profile and promoter analysis of a novel ethylene responsive transcription factor gene GhERF4 from cotton (Gossypium hirstum). Plant physiology and biochemistry 46:46-53. 10.1016/j.plaphy.2007.10.004

Kohler C, Scheid OM, Erilova A. 2010. The impact of the triploid block on the origin and evolution of polyploid plants. Trends in Genetics 26:142-148. 10.1016/j.tig.2009.12.006 
599 Krzywinski M, Schein J, Birol I, Connors J, Gascoyne R, Horsman D, Jones SJ, Marra MA.

600

601

602

603

604

605

606

607

608

609

610

611

612

613

614

615

616

617

618

619

620

621

622

623

624

625

626

627

628

629

630

631

632

633

634

635

636

637

638

2009. Circos: an information aesthetic for comparative genomics. Genome research 19:1639-1645. 10.1101/gr.092759.109

Lescot M, Dehais P, Thijs G, Marchal K, Moreau Y, Peer YVD, Rouze P, Rombauts S. 2002. PlantCARE, a database of plant cis-acting regulatory elements and a portal to tools for in silico analysis of promoter sequences. Nucleic acids research 30:325-327. 10.1093/nar/30.1.325

Letunic I, Bork P. 2016. Interactive tree of life (iTOL) v3: an online tool for the display and annotation of phylogenetic and other trees. Nucleic acids research 44:W242-W245. $10.1093 / \mathrm{nar} / \mathrm{gkw} 290$

Letunic I, Bork P. 2018. 20 years of the SMART protein domain annotation resource. Nucleic acids research 46:D493-D496. 10.1093/nar/gkx922

Li P, Zheng T, Li L, Zhuo X, Jiang L, Wang J, Cheng T, Zhang Q. 2019. Identification and comparative analysis of the CIPK gene family and characterization of the cold stress response in the woody plant Prunus mume. Peerj 7:e6847. 10.7717/peerj.6847

Li X, Tian A, Luo G, Gong Z, Zhang J, Chen S. 2005. Soybean DRE-binding transcription factors that are responsive to abiotic stresses. Theoretical and applied genetics 110:1355-1362. 10.1007/s00122-004-1867-6

Licausi F, Giorgi F, Zenoni S, Osti F, Pezzotti M, Perata P. 2010. Genomic and transcriptomic analysis of the AP2/ERF superfamily in Vitis vinifera. Bmc Genomics 11:719. 10.1186/1471-2164-11-719

Liu L, White MJ, Macrae TH. 1999. Transcription factors and their genes in higher plants functional domains, evolution and regulation. FEBS Journal 262:247-257. 10.1046/j.1432-1327.1999.00349.x

Liu M, Sun W, Ma Z, Zheng T, Huang L, Wu Q, Zhao G, Tang Z, Bu T, Li C, Chen H. 2019. Genome-wide investigation of the AP2/ERF gene family in tartary buckwheat (Fagopyum Tataricum). BMC Plant Biol 19:1-19. 10.1186/s12870-019-1681-6

Liu Y, Chakrabortee S, Li R, Zheng Y, Tunnacliffe A. 2011. Both plant and animal LEA proteins act as kinetic stabilisers of polyglutamine-dependent protein aggregation. FEBS letters 585:630-634. 10.1016/j.febslet.2011.01.020

Loudet 0, Hasegawa P. 2017. Abiotic stress, stress combinations and crop improvement potential. Plant Journal 90:837-838. 10.1111/tpj.13604

Lynch M, Conery JS. 2000. The evolutionary fate and consequences of duplicate genes. Science 290:1151-1155. 10.1126/science.290.5494.1151

Meng X, Li F, Liu C, Zhang C, Wu Z, Chen Y. 2010. Isolation and Characterization of an ERF Transcription Factor Gene from Cotton (Gossypium barbadense L.). Plant Molecular Biology Reporter 28:176-183. 10.1109/TPWRS.2003.818738

Ming R, VanBuren R, Wai CM, Tang H, Schatz M, Bowers J, Lyons E, Wang M-L, Chen J, Biggers E, Zhang J, Huang L, Zhang L, Miao W, Zhang J, Ye Z, Miao C, Lin Z, Wang H, Zhou H, Yim W, Priest H, Zheng C, Woodhouse M, Edger PP, Guyot R, Guo H,

Peer] reviewing PDF | (2020:04:47897:2:0:NEW 12 Aug 2020) 
639

640

641

642

643

644

645

646

647

648

649

650

651

652

653

654

655

656

657

658

659

660

661

662

663

664

665

666

667

668

669

670

671

672

673

674

675

676

677

678

Guo H, Zheng G, Singh R, Sharma A, Min X, Zheng Y, Lee H, Gurtowski J, Sedlazeck F, Harkes. 2015. The pineapple genome and the evolution of CAM photosynthesis. Nature genetics 47:1435-1442. 10.1038/ng.3435

Mittler R. 2006. Abiotic stress, the field environment and stress combination. Trends Plant Sci 11:15-19. 10.1016/j.tplants.2005.11.002

Moose S, Sisco P. 1996. Glossy15, an APETALA2-like gene from maize that regulates leaf epidermal cell identity. Genes \& Development 10:3018-3027. 10.1101/gad.10.23.3018

Muller M, Munnebosch S. 2015. Ethylene Response Factors: A Key Regulatory Hub in Hormone and Stress Signaling. Plant Physiol 169:32-41. 10.1104/pp.15.00677

Nakano T, Suzuki K, Fujimura T, Shinshi H. 2006. Genome-wide analysis of the ERF gene family in Arabidopsis and rice. Plant Physiol 140:411-432. 10.1104/pp.105.073783

Ohmetakagi M, Shinshi H. 1995. Ethylene-inducible DNA binding proteins that interact with an ethylene-responsive element. The Plant cell 7:173-182. 10.1105/tpc.7.2.173

Okamuro J, Caster B, Villarroel R, Montagu MV, Jofuku K. 1997. The AP2 domain of APETALA2 defines a large new family of DNA binding proteins in Arabidopsis. Proceedings of the National Academy of Sciences of the United States of America 94:7076-7081. 10.1073/pnas.94.13.7076

Pan Y, Seymour G, Lu C, Hu Z, Chen X, Chen G. 2012. An ethylene response factor (ERF5) promoting adaptation to drought and salt tolerance in tomato. Plant Cell Reports 31:349-360. 10.1007/s00299-011-1170-3

Peng X, Zhao Y, Li X, Wu M, Chai W, Sheng L, Wang Y, Dong Q, Jiang H, Cheng B. 2015. Genomewide identification, classification and analysis of NAC type gene family in maize. Journal of Genetics 94:377-390. 10.1007/s12041-015-0526-9

Rashid M, He G, Yang G, Hussain J, Xu Y. 2012. AP2/ERF Transcription Factor in Rice: Genome-Wide Canvas and Syntenic Relationships between Monocots and Eudicots. Evolutionary Bioinformatics 8:321-355. 10.4137/EBO.S9369

Rashotte AM, Mason MG, Hutchison CE, Ferreira F, Schaller GE, Kieber JJ. 2006. A subset of Arabidopsis AP2 transcription factors mediates cytokinin responses in concert with a two-component pathway. Proceedings of the National Academy of Sciences of the United States of America 103:11081-11085. 10.1073/pnas.0602038103

Riechmann J, Meyerowitz E. 1998. The AP2/EREBP family of plant transcription factors. Biological Chemistry 379:633-646. 10.1515/bchm.1998.379.6.633

Romanel E, Schrago CG, Counago R, Russo CAM, Alvesferreira M. 2009. Evolution of the B3 DNA Binding Superfamily: New Insights into REM Family Gene Diversification PloS one 4:e5791. 10.1371/journal.pone.0005791

Sakuma Y, Liu Q, Dubouzet J, Abe H, Shinozaki K, Yamaguchi-Shinozaki K. 2002. DNAbinding specificity of the ERF/AP2 domain of Arabidopsis DREBs, transcription factors involved in dehydration- and cold-inducible gene expression. Biochem

Peer] reviewing PDF | (2020:04:47897:2:0:NEW 12 Aug 2020) 
679

680

681

682

683

684

685

686

687

688

689

690

691

692

693

694

695

696

697

698

699

700

701

702

703

704

705

706

707

708

709

710

711

712

713

714

715

716

717

718

Biophys Res Commun 290:998-1009. 10.1006/bbrc.2001.6299

Sang J, Han X, Liu M, Qiao G, Jiang J, Zhuo R. 2013. Selection and Validation of Reference Genes for Real-Time Quantitative PCR in Hyperaccumulating Ecotype of Sedum alfredii under Different Heavy Metals Stresses. PloS one 8:e82927. 10.1371/journal.pone.0082927

Schaefer M, Wanker EE, Andradenavarro MA. 2012. Evolution and function of $\mathrm{CAG}$ /polyglutamine repeats in protein-protein interaction networks. Nucleic acids research 40:4273-4287. 10.1093/nar/gks011

Sharma MK, Kumar R, Solanke AU, Sharma R, Tyagi AK, Sharma AK. 2010. Identification, phylogeny, and transcript profiling of ERF family genes during development and abiotic stress treatments in tomato. Molecular Genetics and Genomics 284:455-475. 10.1007/s00438-010-0580-1

Sharoni AM, Nuruzzaman M, Satoh K, Shimizu T, Kondoh H, Sasaya T, Choi I-R, Omura T, Kikuchi S. 2011. Gene structures, classification and expression models of the AP2/EREBP transcription factor family in rice. Plant and Cell Physiology 52:344-360. $10.1093 / \mathrm{pcp} / \mathrm{pcq} 196$

Stockinger E, Gilmour S, Thomashow M. 1997. Arabidopsis thaliana CBF1 encodes an AP2 domain-containing transcriptional activator that binds to the C-repeat/DRE, a cis-acting DNA regulatory element that stimulates transcription in response to low temperature and water deficit. Proceedings of the National Academy of Sciences of the United States of America 94:1035-1040. 10.1073/pnas.94.3.1035

Su Z, Wang L, Li W, Zhao L, Huang X, Azam SM, Qin Y. 2017. Genome-Wide Identification of Auxin Response Factor (ARF) Genes Family and its Tissue-Specific Prominent Expression in Pineapple ( Ananas comosus ). Tropical Plant Biology 10:86-96. 10.1007/s12042-017-9187-6

Tamura K, Stecher G, Peterson DS, Filipski A, Kumar S. 2013. MEGA6: Molecular Evolutionary Genetics Analysis version 6.0. Molecular biology and evolution 30:2725-2729. 10.1093/molbev/mst197

Tanenbaum D, Goll J, Murphy S, Kumar P, Zafar N, Thiagarajan M, Madupu R, Davidsen T, Kagan L, Kravitz S, Rusch D, Yooseph S. 2010. The JCVI standard operating procedure for annotating prokaryotic metagenomic shotgun sequencing data. Standards in genomic sciences 2:229-237. 10.4056/sigs.651139

Tang H, Bowers J, Wang X, Ming R, Alam M, Paterson A. 2008. Synteny and collinearity in plant genomes. Science 320:486-488. 10.1126/science.1153917

Trapnell C, Roberts A, Goff L, Pertea G, Kim D, Kelley DR, Pimentel H, Salzberg SL, Rinn JL, Pachter L. 2012. Differential gene and transcript expression analysis of RNA-seq experiments with TopHat and Cufflinks. Nature protocols 7:562-578. $10.1038 /$ nprot.2012.016

Uversky VN. 2015. The intrinsic disorder alphabet. III. Dual personality of serine. Intrinsically Disordered Proteins 3:e1027032. 10.1080/21690707.2015.1027032 
719

720

721

722

723

724

725

726

727

728

729

730

731

732

733

734

735

736

737

738

739

740

741

742

743

744

745

746

747

748

749

750

751

752

753

754

755

756

757

758

Vernie T, Moreau S, Billy FD, Plet J, Combier J, Rogers C, Oldroyd GED, Frugier F, Niebel A, Gamas P. 2008. EFD Is an ERF transcription factor involved in the control of nodule number and differentiation in Medicago truncatula. The Plant cell 20:2696-2713. 10.1105/tpc.108.059857

Vision T, Brown DG, Tanksley SD. 2000. The origins of genomic duplications in Arabidopsis. Science 290:2114-2117. 10.1126/science.290.5499.2114

Voorrips RE. 2002. MapChart: software for the graphical presentation of linkage maps and QTLs. Journal of Heredity 93:77-78. 10.1093/jhered/93.1.77

Wang W. 2015. The Molecular Detection of Corynespora Cassiicola on Cucumber by PCR Assay Using DNAman Software and NCBI. International Conference on Computer and Computing Technologies in Agriculture. p 248-258.

Xie T, Chen C, Li C, Liu J, Liu C, He Y. 2018. Genome-wide investigation of WRKY gene family in pineapple: evolution and expression profiles during development and stress. Bmc Genomics 19:1-18. 10.1186/s12864-018-4880-x

Xu K, Xu X, Fukao T, Canlas PE, Maghirangrodriguez R, Heuer S, Ismail A, Baileyserres J, Ronald PC, Mackill DJ. 2006. Sub1A is an ethylene-response-factor-like gene that confers submergence tolerance to rice. Nature 442:705-708. 10.1038/nature04920

Yu Z, Li J, Yang C, Hu W, Wang L, Chen X. 2012. The jasmonate-responsive AP2/ERF transcription factors AaERF1 and AaERF2 positively regulate artemisinin biosynthesis in Artemisia annua L. Molecular plant 5:353-365. 10.1093/mp/ssr087

Zeng R, Zhu J, Xu S, Du G, Guo H, Chen J, Zhang Z, Xie L. 2020. CymbidiumUnreduced Male Gamete Formation in and Its Use for Developing Sexual Polyploid Cultivars. Frontiers in plant science 11:558. 10.3389/fpls.2020.00558

Zhang C, Shangguan L, Ma R, Sun X, Tao R, Guo L, Korir NK, Yu M. 2012. Genome-wide analysis of the AP2/ERF superfamily in peach (Prunus persica). Genetics and Molecular Research 11:4789. 10.4238/2012.0ctober.17.6

Zhang G, Ming C, Li L, Xu Z, Chen X, Guo J, Ma Y. 2009. Overexpression of the soybean GmERF3 gene, an AP2/ERF type transcription factor for increased tolerances to salt, drought, and diseases in transgenic tobacco. Journal of experimental botany 60:3781-3796. 10.1093/jxb/erp214

Zhang M, Liu Y, He Q, Chai M, Huang Y, Chen F, Wang X, Liu Y, Cai H, Qin Y. 2020. Genome-wide investigation of calcium-dependent protein kinase gene family in pineapple: evolution and expression profiles during development and stress. Bmc Genomics 21:72. 10.1186/s12864-020-6501-8

Zhang M, Liu Y, Shi H, Chai M, He Q, Yan M, Cao D, Zhao L, Cai H, Qin Y. 2018. Evolutionary and expression analyses of soybean basic Leucine zipper transcription factor family. Bmc Genomics 19:1-14. 10.1186/s12864-018-4511-6

Zhu Y, Wu N, Song W, Yin G, Qin Y, Yan Y, Hu Y. 2014. Soybean (Glycine max) expansin gene superfamily origins: segmental and tandem duplication events followed by divergent selection among subfamilies. BMC Plant Biol 14:93. 10.1186/1471-2229-

PeerJ reviewing PDF | (2020:04:47897:2:0:NEW 12 Aug 2020) 
759

760

761

762

763

764

765

766

767

768

14-93

Zhuang J, Cai B, Peng R, Zhu B, Jin X, Xue Y, Gao F, Fu X, Tian Y, Zhao W, Qiao Y, Zhang Z, Xiong A, Yao Q. 2008. Genome-wide analysis of the AP2/ERF gene family in Populus trichocarpa. Biochem Biophys Res Commun 371:468-474. 10.1016/j.bbrc.2008.04.087

Zhuang J, Peng R, Cheng Z, Zhang J, Cai B, Zhang Z, Gao F, Zhu B, Fu X, Jin X, Chen J, Qiao Y, Xiong A, Yao Q. 2009. Genome-wide analysis of the putative AP2/ERF family genes in Vitis vinifera. Scientia Horticulturae 123:73-81.

10.1016/j.scienta.2009.08.002 
Figure 1

The evolutionary relationship of the ERFs.

(A) Unrooted phylogenetic tree representing relationship among AP2/ERF genes between pineapple and Arabidopsis; (B) Phylogenetic analysis of ERF proteins from pineapple and Arabidopsis. The genes in pineapple are marked in red, while those in Arabidopsis are marked in black. The pineapple DREB proteins are classified into five groups: I, II, III, IV and V.

A

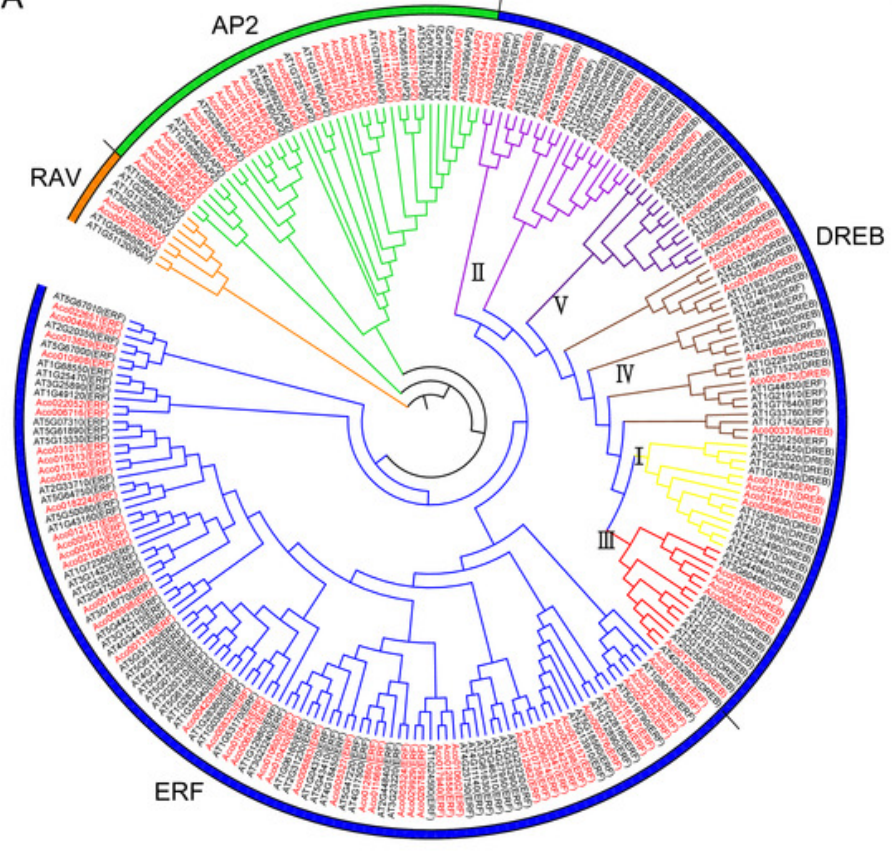

B

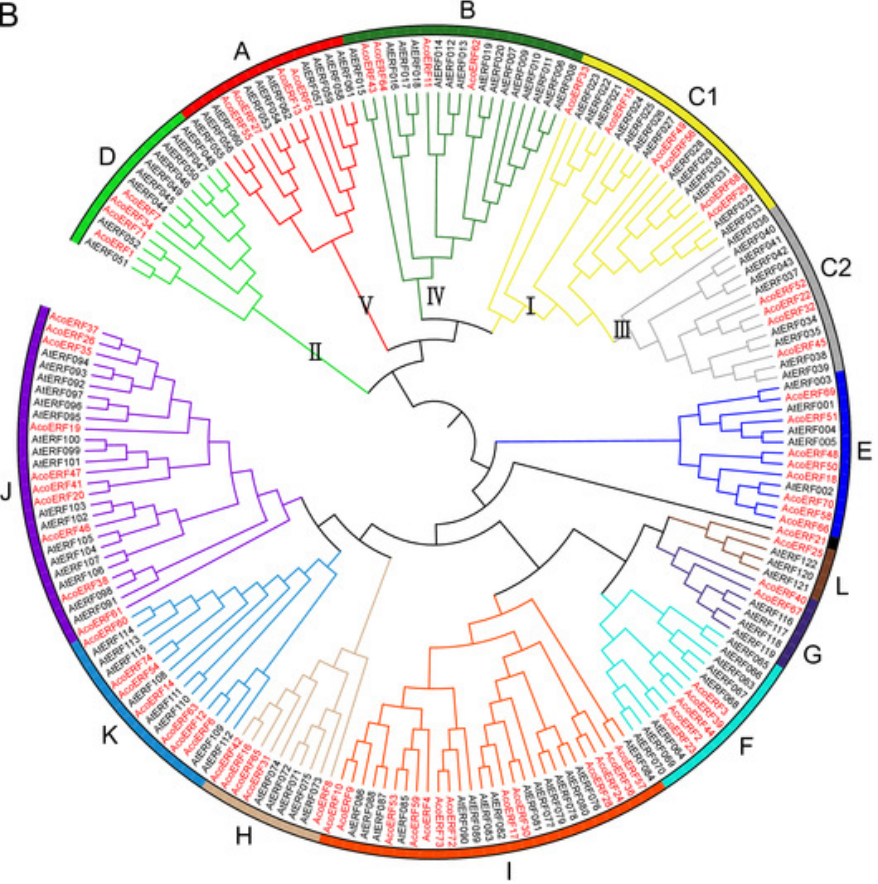


Figure 2

The exon-intron structure of AcoERF genes based on the evolutionary relationship.

The yellow round-corner rectangle represents exons, the black shrinked line represents introns, and the blue round-corner rectangle represents UTR. 


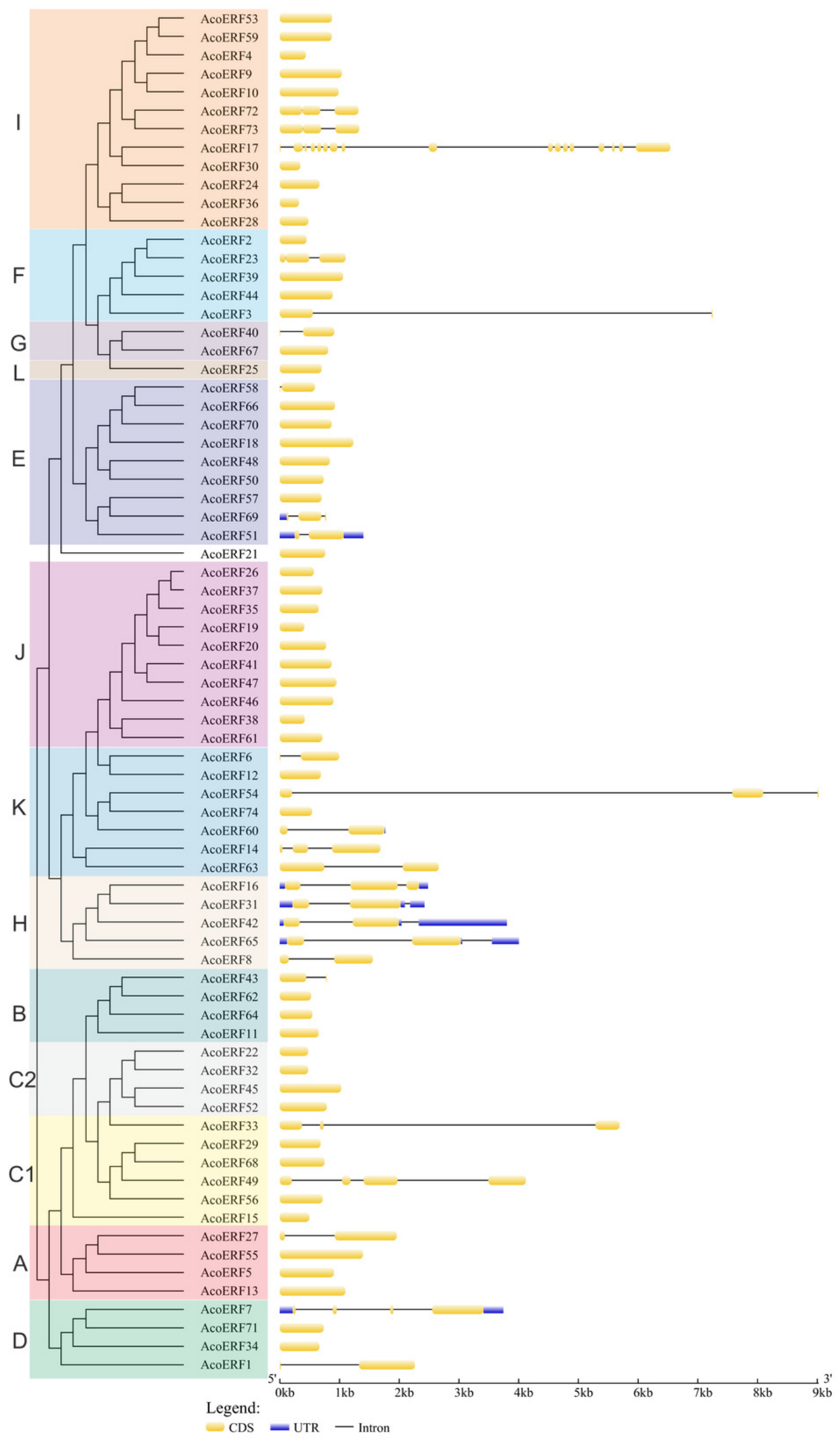


Figure 3

Motif distribution of ACOERF proteins.

The conserved motifs in the AcOERF proteins were identified with MEME software. Grey lines denote the non-conserved sequences, and each motif is indicated by a colored box numbered at the bottom. The length of motifs in each protein was presented proportionally.

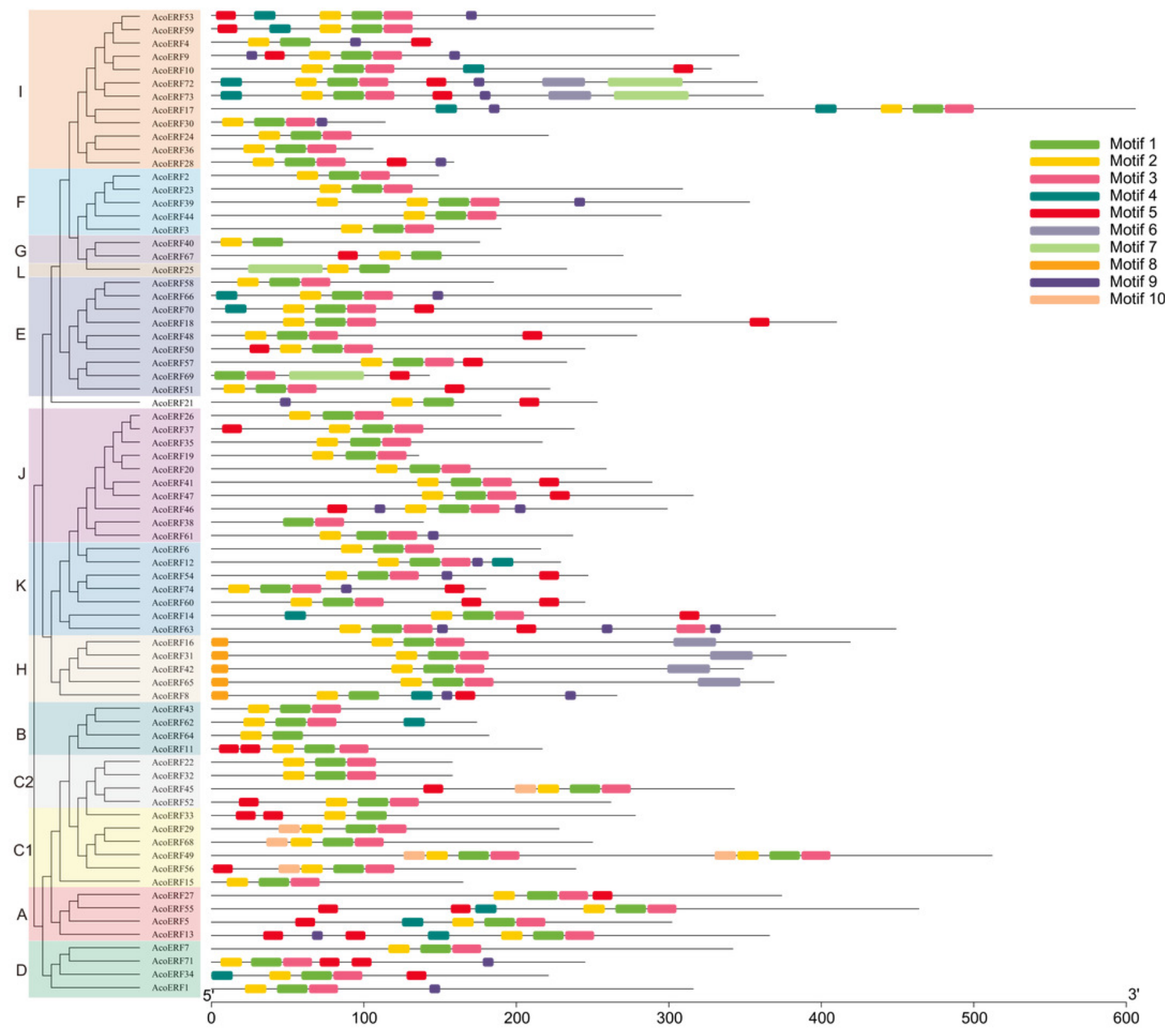


Figure 4

Schematic representations for the chromosomal distribution and interchromosomal relationships of pineapple $E R F$ genes.

The red lines indicate duplicated ERF gene pairs, and the chromosome number is indicated at the bottom of each chromosome. 


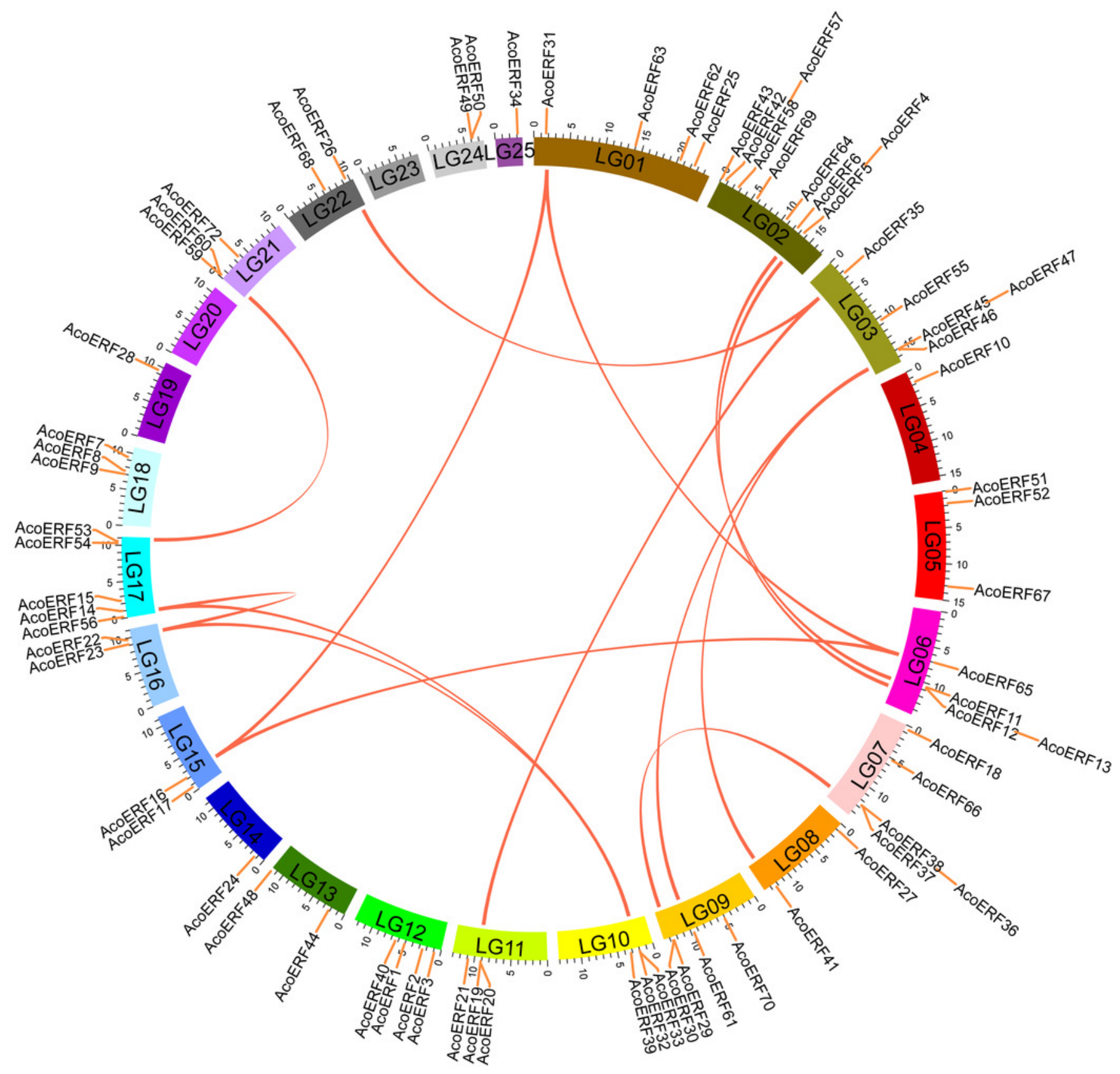


Figure 5

Synteny analysis of $E R F$ genes between pineapple and two representative plant species.

(A) Synteny analysis of ERF genes between pineapple and Arabidopsis; (B) Synteny analysis of ERF genes between pineapple and rice. Gray lines in the background indicate the collinear blocks within pineapple and other plant genomes, while the red lines highlight the syntenic ERF gene pairs.

A $\begin{array}{lllllllllllllll}1 & 2 & 3 & 4 & 5 & 6 & 7 & 8 & 9 & 10 & 11 & 12 & 13 & 14 & 1516171819202122232425\end{array}$
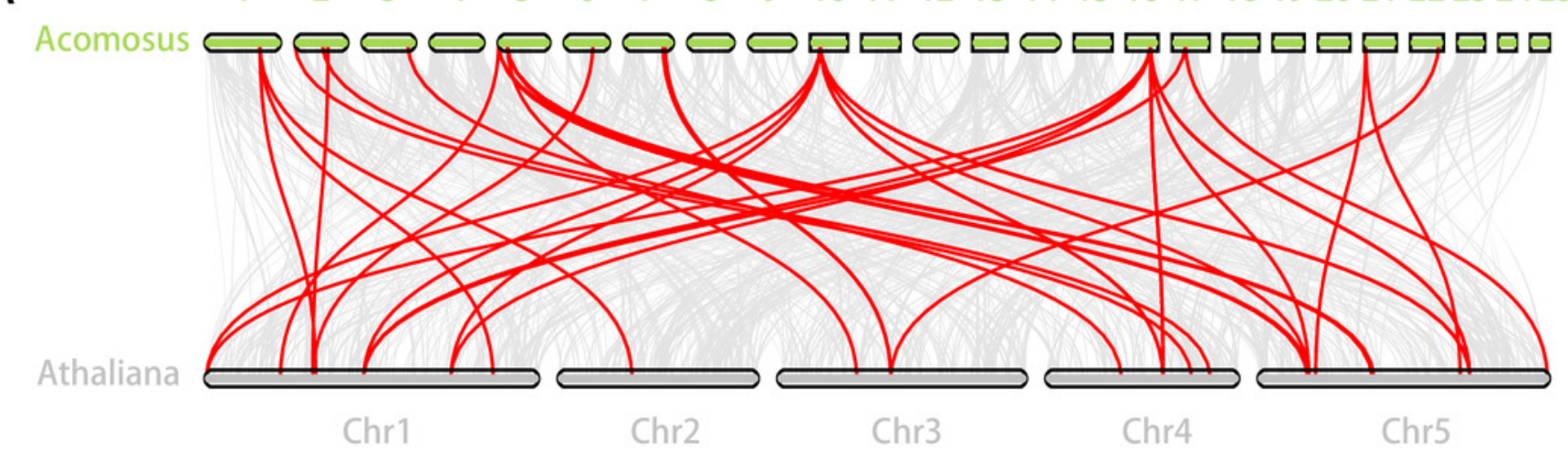

B

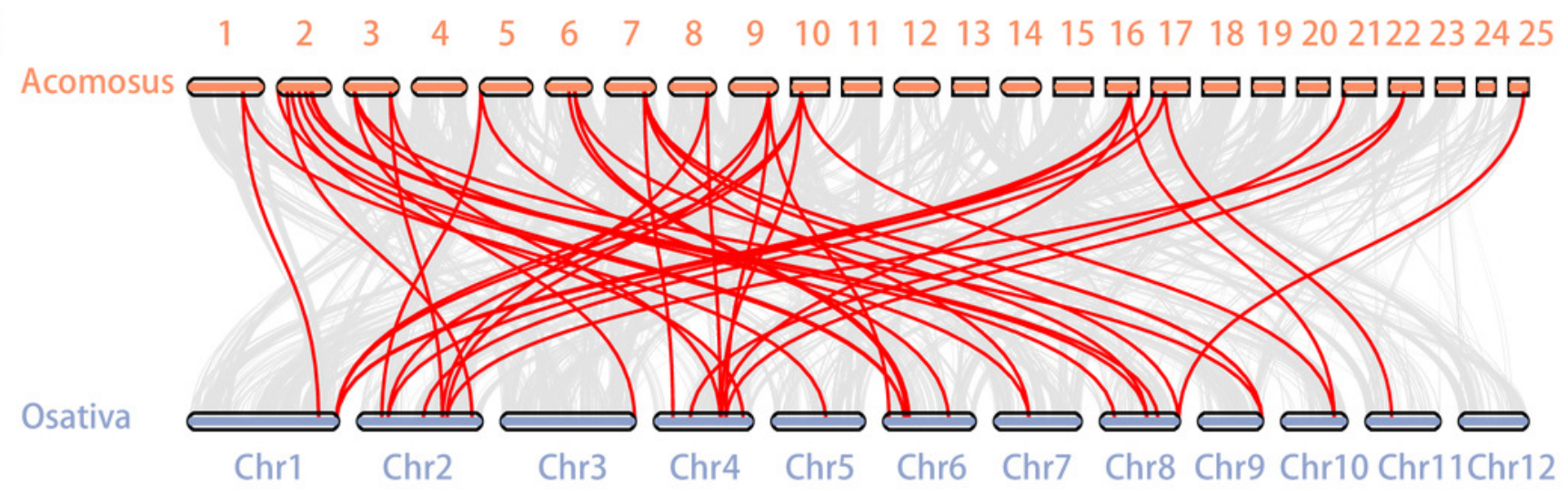


Figure 6

Expression profiles of 74 AcoERF genes in different tissues and stages of pineapple.

Different colors in map represent gene transcript abundance values as shown in bar by the side of the figure. 


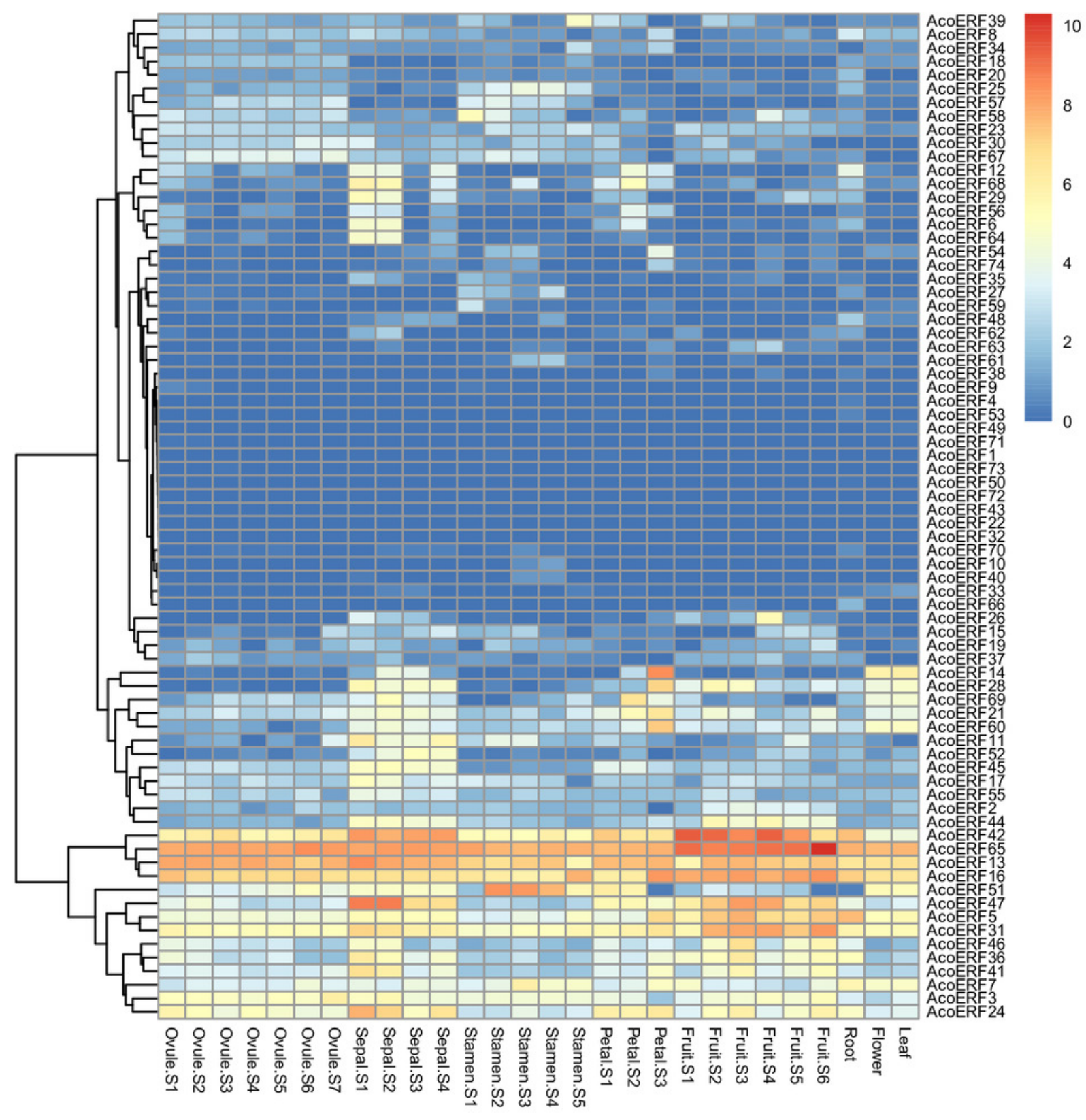


Figure 7

Expression profile of 9 selected AcoERF genes in response to cold stress treatment.

Error bars indicate the standard deviation. Asterisks on top of the bars indicating statistically significant differences between the stress and counterpart controls $\left({ }^{*} P<0.05,{ }^{* * P}<0.01\right)$.

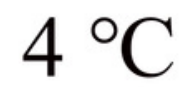

AcoERF7

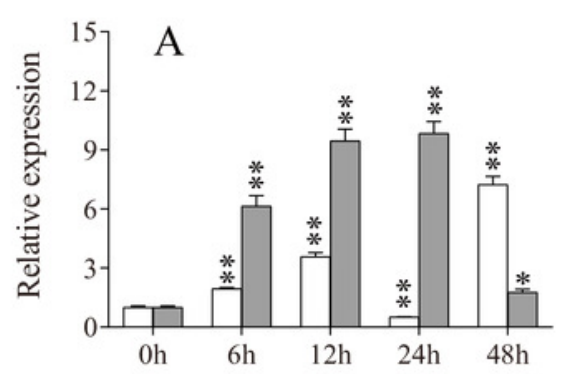

AcoERF36

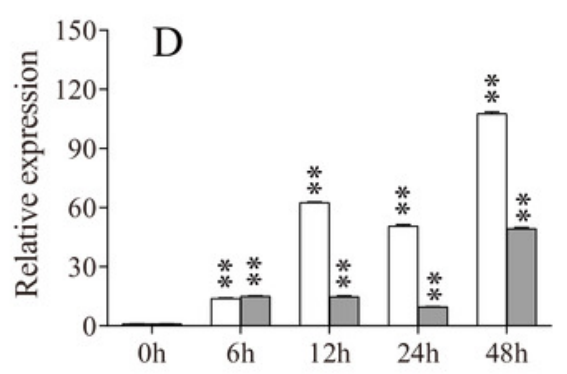

AcoERF60

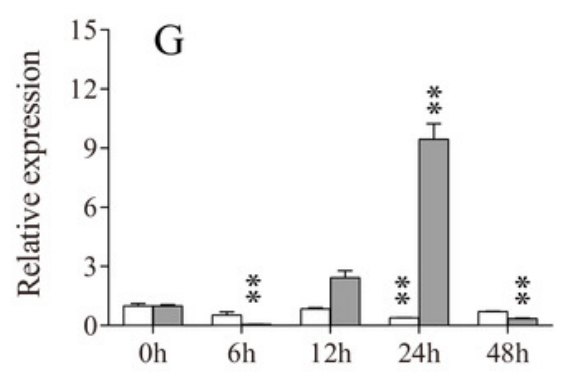

AcoERF13

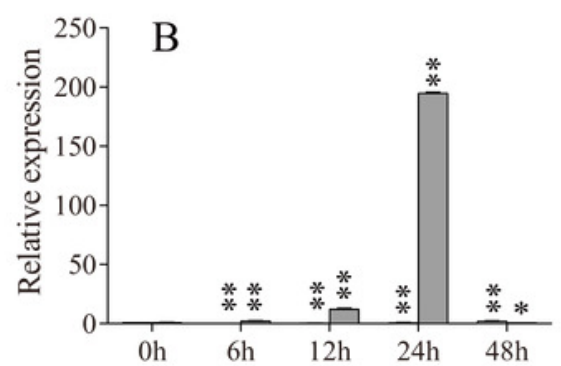

AcoERF42

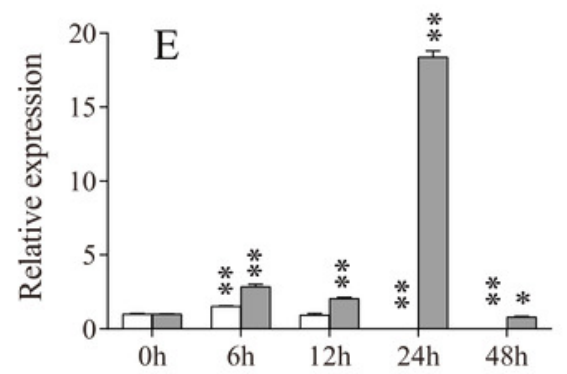

AcoERF65

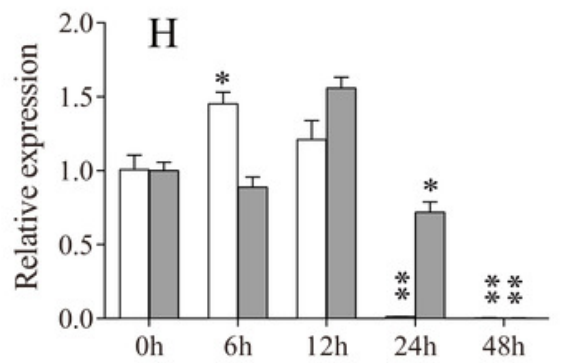

$\square$ Root $\square$ Leaf

AcoERF16

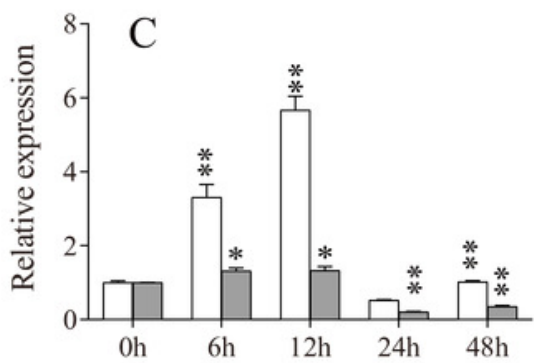

AcoERF45

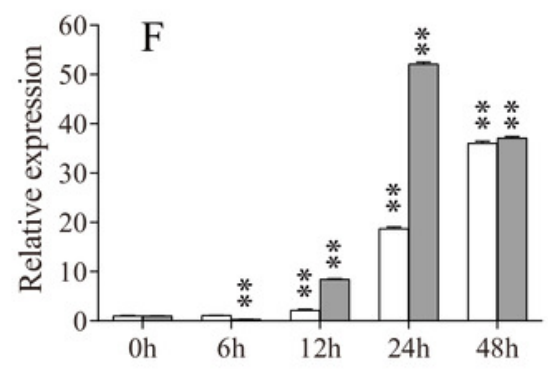

AcoERF67

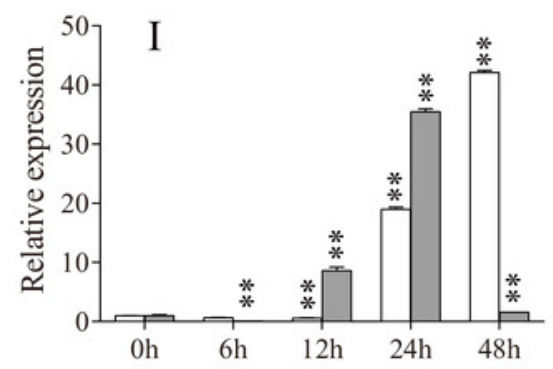


Figure 8

Expression profile of 9 selected ACOERF genes in response to drought stress treatment.

Error bars indicate the standard deviation. Asterisks on top of the bars indicating statistically significant differences between the stress and counterpart controls $\left({ }^{*} \mathrm{P}<0.05,{ }^{* * P}<0.01\right)$.

\section{Mannitol}

AcoERF7

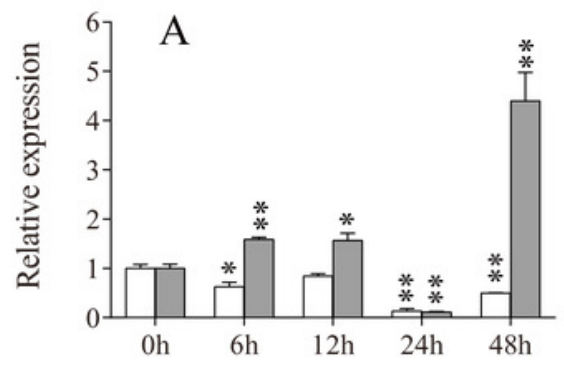

AcoERF36

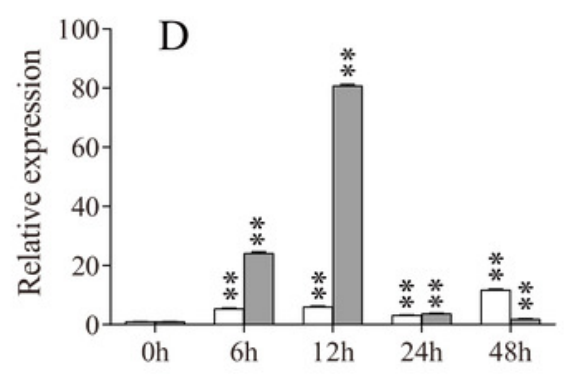

AcoERF60

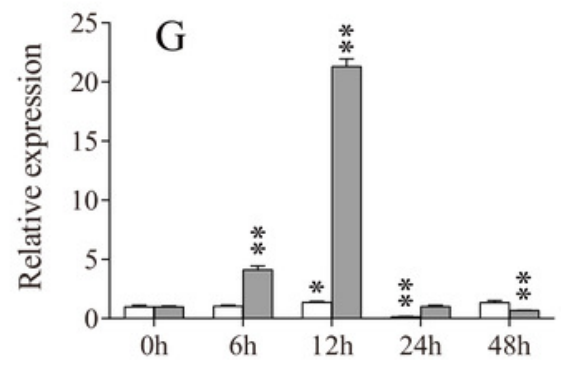

AcoERF13

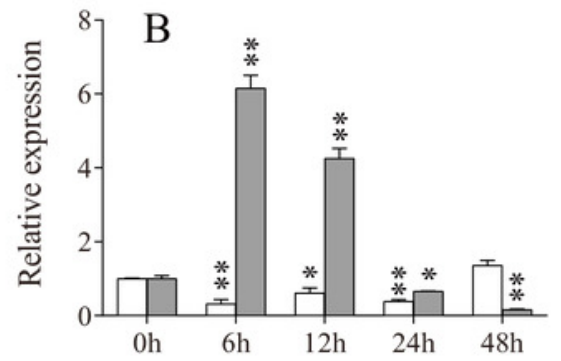

AcoERF42

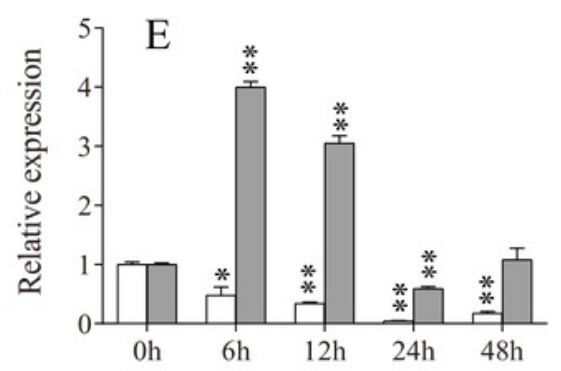

AcoERF65

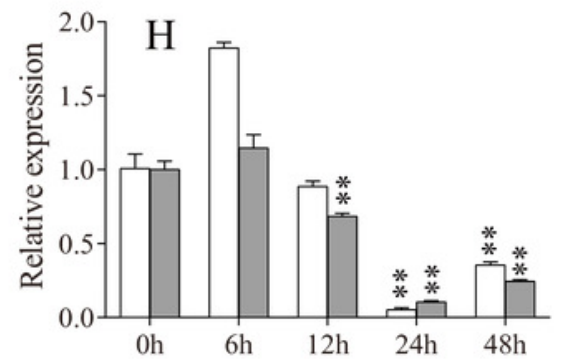

$\square$ Root $\square$ Leaf AcoERF16

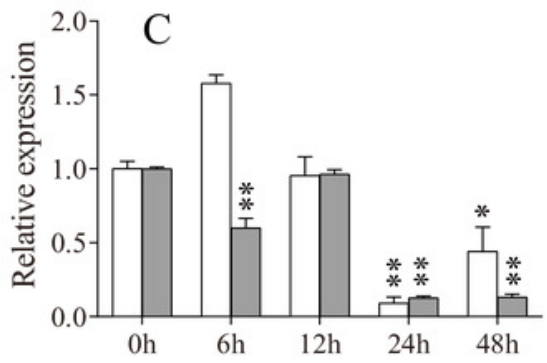

AcoERF45

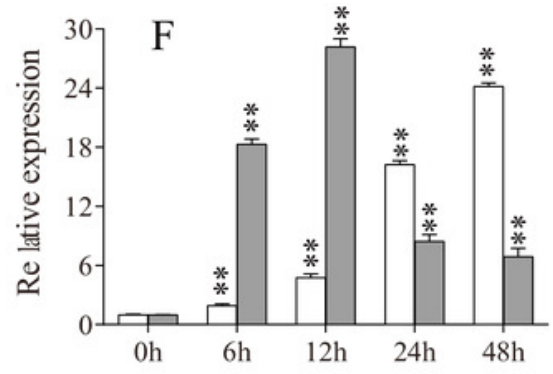

AcoERF67

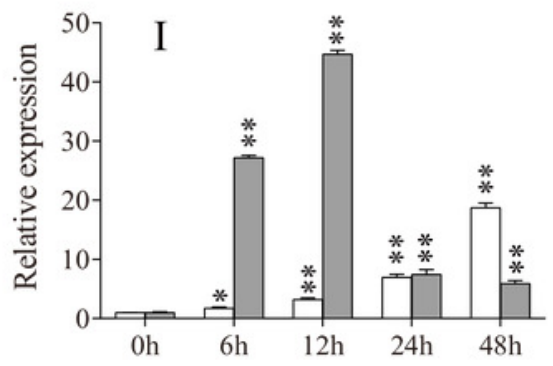


Figure 9

Expression profile of 9 selected AcoERF genes in response to salt stress treatment.

Error bars indicate the standard deviation. Asterisks on top of the bars indicating statistically significant differences between the stress and counterpart controls $\left({ }^{*} \mathrm{P}<0.05,{ }^{* * P}<0.01\right)$.

\section{$\mathrm{NaCl}$}

AcoERF7

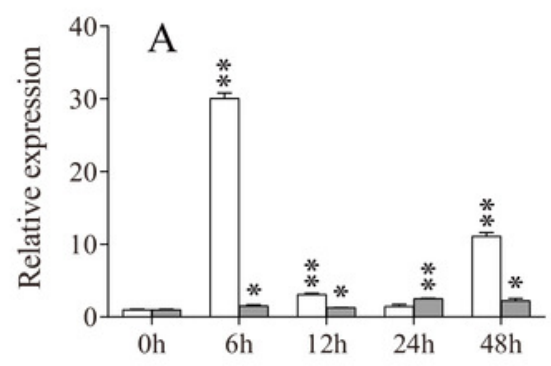

AcoERF36

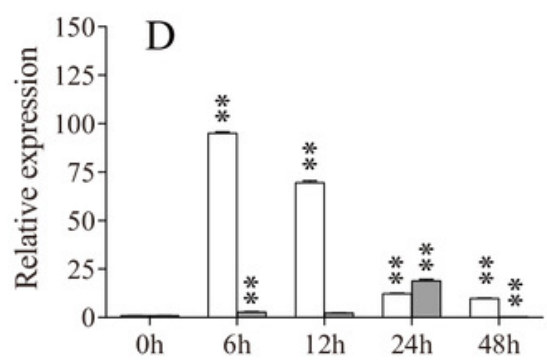

AcoERF60

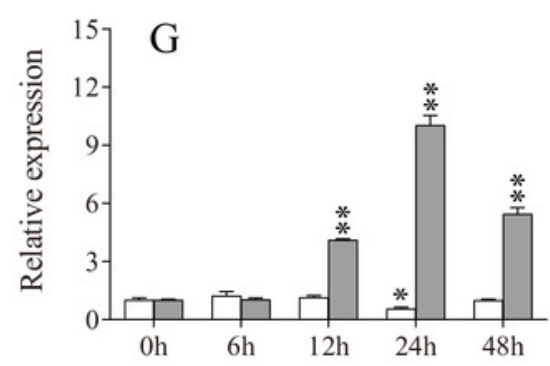

AcoERF13

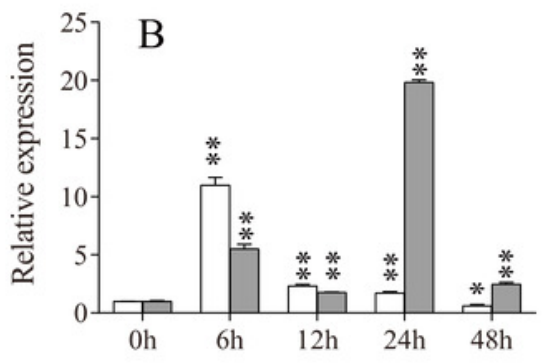

AcoERF42

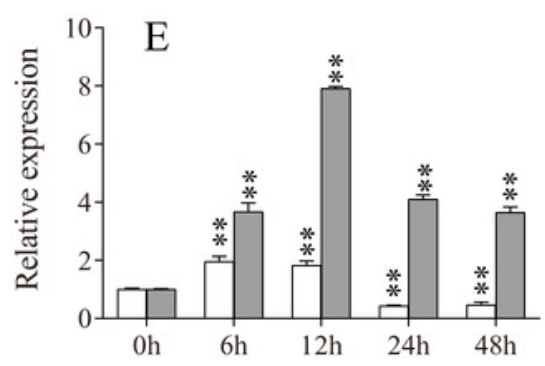

AcoERF65

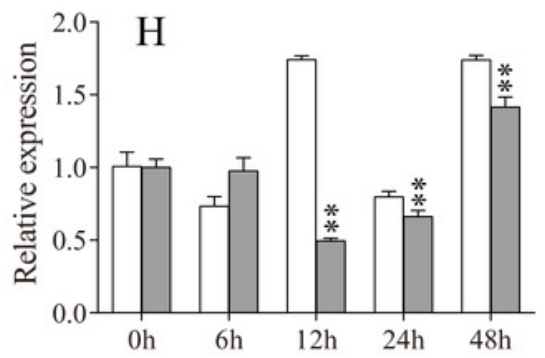

$\square$ Root $\square$ Leaf

AcoERF16

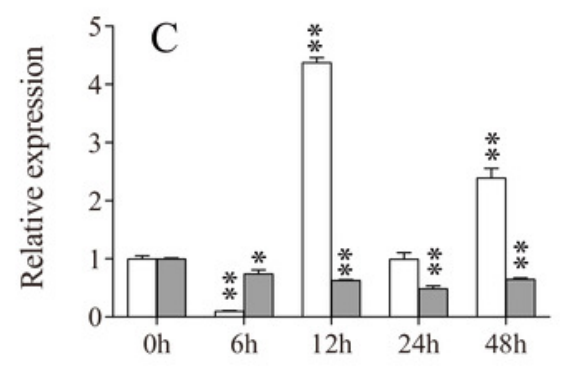

AcoERF45

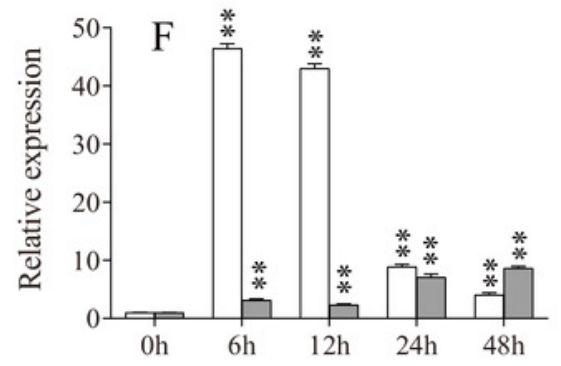

AcoERF67

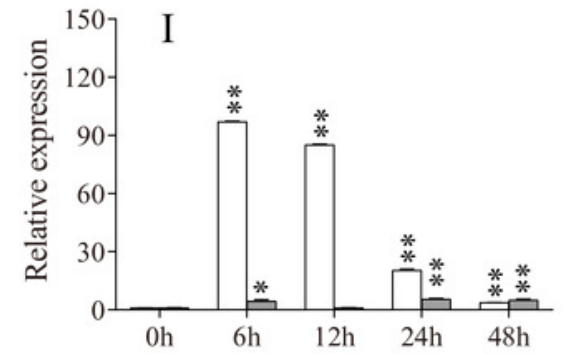

